\title{
Weighted Trudinger-Type Inequalities
}

\section{Stephen M. Buckley $\mathscr{G}$ Julann O'Shea}

\begin{abstract}
We establish sharp inequalities of Trudinger-type (with non-standard Young functions) on general domains $\Omega$ with respect to measures $\nu, \mu$. Our results are new even when $\Omega$ is a Euclidean ball, and $\nu, \mu$ are defined in terms of powers of distance to the boundary. In the Euclidean case, sharpness results are proved for the Young functions involved and the class of domains considered. New characterizations of QHBC domains are given.
\end{abstract}

\section{INTRODUCTION}

The (unweighted) Trudinger inequality [32] on an open ball in $\mathbb{R}^{n}$ is a substitute for the Sobolev-Poincaré inequality in the limiting case where the exponent of the gradient equals the dimension $n$ (we assume $n>1$ throughout this paper). Weighted Poincaré-type inequalities have been extensively studied - see, for example, [6], [9], [11], [12], [13], [14], [15], [20], [21], [23]-but limiting cases of these inequalities have received scant attention (we know only of [4] and [26], both of which consider only special families of weights). Here, we shall investigate Trudinger-type inequalities for rather general pairs of weights (or measures) on fairly general domains. We prove sharp Trudinger-type inequalities and also give a weighted generalization of the "Trudinger implies QHBC" result of [3]. We mainly restrict our attention to the Euclidean setting, although we shall consider imbeddings in more general metric measure spaces in Section 6 .

One of the main lessons of this paper is that these Trudinger-type inequalities are governed by balance conditions in much the same way that Poincaré-type inequalities are governed by the balance condition of Chanillo-Wheeden [9]. Despite this similarity in function, the balance conditions for Poincaré-type and Trudinger-type inequalities are quite different in form. This is not surprising since, for example, John domains are the right class for the Sobolev-Poincaré inequality, while QHBC domains are the right class for Trudinger's inequality; see [3]. QHBC domains, sometimes known as Hölder domains, are defined in terms 
of the quasihyperbolic metric (see Section 2), and include the more well-known class of John domains. For now let us note that a simply-connected planar domain $\Omega$ is QHBC if and only if the Riemann mapping from the unit disk $\mathbb{D}$ to $\Omega$ has a Hölder continuous extension to $\overline{\mathbb{D}}$.

As in the unweighted case, our Trudinger-type inequalities involve Orlicz norms, but the best possible Orlicz function depends on the weights involved, and not just on the dimension. By an Orlicz function, we mean a homeomorphism $\varphi:[0, \infty) \rightarrow[0, \infty)$. If $\mu$ is a positive measure on a Euclidean domain $\Omega$, we define the Orlicz "norm"

$$
\|f\|_{\varphi(L)(\Omega, \mu)}=\inf \left\{t>0 \mid \int_{\Omega} \varphi\left(\frac{|f(x)|}{t}\right) d \mu(x) \leq 1\right\}
$$

As is well known, one needs to assume that $\varphi$ is a Young function, i.e. a convex Orlicz function, in order for this to be a genuine norm. If $\mu$ is not specified, it is taken to be Lebesgue measure. We say that a bounded domain $\Omega \subset \mathbb{R}^{n}$ supports a Trudinger inequality if there exists a constant $C$ such that

$$
\text { for all } u \in \operatorname{Lip}(\Omega): \quad\left\|u-u_{\Omega}\right\|_{\varphi(L)(\Omega)} \leq C\|\nabla u\|_{L^{n}(\Omega)},
$$

where $u_{\Omega}$ is the Lebesgue average of $u$ on $\Omega$ and $\varphi(x)=\exp \left(x^{n /(n-1)}\right)-1$.

Trudinger [32] proved such an inequality for domains with a uniform interior cone condition. Later, Smith and Stegenga [31] showed that QHBC domains support a Trudinger inequality, with a constant dependent only on $n$ and the QHBC constant of $\Omega$. Conversely, it was shown in [3] that for many classes of domains (e.g. finitely-connected plane domains or domains quasiconformally equivalent to a uniform domain) $\Omega$ must be $\mathrm{QHBC}$ if it satisfies a Trudinger inequality.

Let us mention some notational conventions that we shall use. Except in Section 6 (where we consider more general spaces), $\Omega$ is a bounded domain in $\mathbb{R}^{n}$ and $\delta(x)=\operatorname{dist}(x, \partial \Omega), x \in \Omega$. We denote by $u_{S, \mu}$ or $f_{S} u d \mu$ the average of a function $u$ on a set $S$ with respect to a measure $\mu$; we also write $u_{S, w}$, if $d \mu=w d x$, and we simply write $u_{S}$ or $f_{S} u$ if $\mu$ is Lebesgue measure. If $u$ is non-negative, we sometimes write $u(S)$ in place of $\int_{S} u$. For any exponent $1<p<\infty$, we write $p^{\prime}=p /(p-1)$.

Let $s \geq 0$ and let $\varphi_{s}(x)=\exp \left(x^{(n+s)^{\prime}}\right)-1$ (note that $\varphi_{s}$ is a Young function). Suppose that $\nu, \mu$ are positive Borel measures on $\Omega$ and let us write $\left|\nabla^{+} u\right|(x)=\limsup _{y \rightarrow x}|u(x)-u(y)| /|x-y|$. If

$$
\begin{aligned}
& \exists C_{0}<\infty: \quad \text { for all } u \in \operatorname{Lip}(\Omega), \\
& \quad\left\|u-u_{\Omega, \nu}\right\|_{\varphi_{s}(L)(\Omega, \nu)} \leq C_{0}\left\|\left|\nabla^{+} u\right|\right\|_{L^{n+s}(\Omega, \mu)},
\end{aligned}
$$


we say that $(\Omega, \nu, \mu)$ supports an $s$-Trudinger inequality; if $d \nu=v d x, d \mu=w d x$, we may simply say that $(\Omega, v, w)$ supports an $s$-Trudinger inequality.

If $u$ is differentiable $\mu$-almost everywhere, we may replace $\left|\nabla^{+} u\right|$ by $|\nabla u|$ on the right-hand side of (1.1); in particular we do this if either $u \in C^{1}(\Omega)$ or $\mu$ is absolutely continuous with respect to Lebesgue measure. Note also that $\left|\nabla^{+} u\right|$ is an "upper gradient" of $u$; see [21, Proposition 9.2].

Ideally we would like to classify all triples that support an $s$-Trudinger inequality; without some restrictions, however, this is a hopelessly optimistic undertaking. We shall need three types of "technical assumptions" at various points. First we need local restrictions on $\nu, \mu$ (such as local doubling and $A_{1}$ conditions). As with Poincaré inequalities, easy examples show that some such restrictions are necessary; these conditions are rather mild because they do not restrict the boundary behavior of $\nu, \mu$, and because we allow rather general sets (if we want to study, for example, local doubling measures which are nasty in the vicinity of an interior point, we simply remove that point from the domain). The second type of restriction is that one or both measures are assumed to be "strong doubling"; this restricts their boundary behavior, but in a rather mild way. Finally, we need various geometric restrictions on $\Omega$. All except one of our imbedding results are valid for all QHBC domains. The exceptional result says that an $s$-Trudinger inequality implies a global balance condition; here, we need to assume that $\Omega$ has a slice property (and so this result is true for John domains, finitely-connected plane domains, quasiconformal images of uniform domains, etc.).

Ignoring technical assumptions, our main imbedding result, Theorem 3.10, roughly says the following (at least when $s>0$ ): if $\Omega$ is $\mathrm{QHBC}$, then $(\Omega, \nu, \mu)$ supports an $s$-Trudinger inequality if and only if $\nu, \mu$ satisfy a certain balance condition (which in particular implies that " $d \mu$ is not much smaller than $\delta^{s} d x "$ ).

These imbeddings are naturally of greater significance if we can show that the Orlicz function $\varphi_{s}$ is sharp. We shall see that $d \mu$ must, in some sense, look like $\delta^{s} d x$ in order for the imbedding to be both valid and sharp. If, for example, $d \mu=w(x) d x$, where $w(x) \leq C|x-z|^{s}$ for some $z \in \partial \Omega$, then $\varphi_{s}$ usually cannot be improved. Additionally, if $w \leq C \delta^{s}$, then $s$-Trudinger imbeddings usually force $\Omega$ to be QHBC. The word "usually" is employed above because various technical assumptions are required, e.g. $\nu$ should have "polynomial decay rate" near the boundary. The " $s$-Trudinger implies QHBC" result, which generalizes one of the main results of [3], also requires that we assume that the domain has a "weak slice property." This is a notable improvement over the unweighted result in [3] where $\Omega$ is assumed to have a slice property, since QHBC domains must have a weak slice property, but may not have a slice property. Thus we get new characterizations of the QHBC condition.

The paper [4], which uses very different methods to prove some imbeddings for "power weights" (i.e. powers of $\delta(x)$ ), was the primary motivation for this much wider investigation. Consequently, we shall pay special attention to imbeddings for power weights. As such weights automatically satisfy most of our 
technical assumptions, we get more simply stated results.

We pause to state the main imbedding theorem for power weights; it will follow easily from several of the more general results. The (non-sharp) case $t=s$ of this theorem was proved in [4] for balls (and for QHBC domains if $t=s \in \mathbb{N}$ ); see also [26] and [19] for related results.

Theorem 1.2. Suppose that $\Omega \subset \mathbb{R}^{n}$ is a QHBC domain, $s \geq 0, d \mu=$ $\delta^{s} d x$. Let $t_{0}=\inf \left\{t \in \mathbb{R}: \int_{\Omega} \delta^{t} d x<\infty\right\}$, so that $-1 \leq t_{0}<0$. Then $\left(\Omega, \delta^{t}, \delta^{s}\right)$ supports an $s$-Trudinger inequality if $t>t_{0}$, but not if $t<t_{0}$. Furthermore, the Orlicz function $\varphi_{s}$ is sharp, i.e. if $d \nu=\delta^{t} d x$ for any $t \in \mathbb{R}$, then (1.1) becomes false if we replace $\varphi_{s}$ by any Orlicz function $\psi$ such that $\psi(c x) / \varphi_{s}(x) \rightarrow \infty(x \rightarrow$ $\infty)$ for all $c>0$.

The above imbeddings are limiting cases of known Poincaré-type imbeddings. For instance, if $\Omega$ is a ball, $X=L^{(n+s) p /(n+s-p)}\left(\Omega, \delta^{s} d x\right), Y=L^{p}\left(\Omega, \delta^{s} d x\right)$, and $s=t$, then $\left[9\right.$, Theorem 1.3] tells us that $\left\|u-u_{\Omega, \delta^{s}}\right\|_{X} \leq C\|\nabla u\|_{Y}$ (see also [5]). One would therefore expect some sort of exponential integrability of $\left|u-u_{\Omega, \delta^{s}}\right|$ when $p=n+s$, but the precise nature of the sharp limiting imbedding is not obvious.

The rest of this paper is organized as following. We discuss some background material in Section 2, state our main imbedding results in Section 3, and prove them in Section 4. In Section 5, we prove sharpness results and, in Section 6, we discuss Trudinger-type imbeddings on Carnot-Carathéodory and other metric measures spaces.

\section{Preliminary Definitions AND Results}

We begin with a pair of lemmas. The first is a well-known Hölder imbedding; for a proof in the case of balls, see [1]. The second is a version of the Whitney decomposition, as given in [29]. Below, $L^{1, p}(\Omega)$ is the space of locally integrable functions whose distributional gradients belong to $L^{p}(\Omega)$.

Lemma 2.1. Let $p>n$ and suppose $u \in L^{1, p}(Q)$, where $Q \subset \mathbb{R}^{n}$ is an open cube. Then $u$ has a continuous representative, and there is a constant $C=C(p, n)>0$, such that for all $x, y \in Q$,

$$
|u(x)-u(y)| \leq C|x-y|^{1-n / p}\|\nabla u\|_{L^{p}(Q)} .
$$

Lemma 2.2. Given $A \geq 1$, there is $C=C(A, n)$ such that if $\Omega$ is a proper subdomain of $\mathbb{R}^{n}$, then $\Omega=\bigcup_{j} \bar{Q}_{j}$, where the $Q_{j}$ are disjoint open cubes satisfying

(i) $5 A \leq \frac{\operatorname{dist}\left(Q_{j}, \partial \Omega\right)}{\operatorname{diam} Q_{j}} \leq 15 A$.

(ii) $\sum_{j} \chi_{A Q_{j}} \leq C \chi_{\Omega}$. 
Here and throughout the rest of the paper, $r Q$ and $r B$ denote the concentric dilate of a cube $Q$ or ball $B$ by a factor $r>0$. We denote by $\mathcal{W}(\Omega)$ "the" Whitney decomposition of a domain $\Omega$, by which we mean a collection of such cubes $\left\{Q_{j}\right\}$ in the case $A=20$ (the definition of $\mathcal{W}(\Omega)$ will be somewhat modified for general spaces in Section 6). We may also assume that all Whitney cubes are dyadic cubes, and so their sidelengths are powers of 2 .

If $Q$ is a Whitney cube, we denote its sidelength by $\delta_{Q}$ (note that $\delta_{Q}$ and $\delta(x)$ are within some fixed factor of one another whenever $x \in Q$ ). In proofs, $C$ denotes any positive constant whose exact value does not matter (and can change from line to line); we write $A \lesssim B$ (or $B \gtrsim A$ ) if $A \leq C B$, and $A \approx B$ if $A \lesssim B \lesssim A$.

The quasihyperbolic length of a rectifiable path $\gamma \subset \Omega$, is defined by $k_{\Omega}(\gamma)=$ $\int_{\gamma} \delta(x)^{-1} d s$. We then define $k_{\Omega}(x, y)$, the quasihyperbolic distance between $x, y \in$ $\Omega$, to be the infimum of $k_{\Omega}(\gamma)$, as $\gamma$ ranges over all paths linking $x$ and $y$. For any pair of points $x, y$, there always exists a quasihyperbolic geodesic, i.e. a path $\gamma_{x, y}$ such that $k_{\Omega}(x, y)=k_{\Omega}\left(\gamma_{x, y}\right)$; see [18]. Clearly, $1+k_{\Omega}(\gamma)$ is greater than some fixed factor times the number of Whitney cubes through which $\gamma$ passes. For a geodesic path this inequality can be reversed, since we can replace any path by an approximating polygonal path with the same entry and exit points on the closure of each Whitney cube. Thus $1+k_{\Omega}(x, y)$ is, to within a fixed factor, equal to the number of Whitney cubes through which $\gamma_{x, y}$ passes.

We next define some geometric conditions on Euclidean domains that will be needed later. First we say that a bounded domain $\Omega$ is a John domain with respect to $x_{0} \in \Omega$ if it satisfies the following "carrot" condition: there exists a constant $C \geq 1$ such that for all $x \in \Omega$, there is a path $\gamma=\gamma_{x}:[0, l] \rightarrow \Omega$ parametrized by arclength such that $\gamma(0)=x, \gamma(l)=x_{0}$, and $\delta(\gamma(t)) \geq t / C$. We call $x_{0}$ the John center of $\Omega, \gamma$ the John path for $x$, and we define the John constant of $\Omega$ (with respect to $x_{0}$ ) to be the smallest $C$ for which the above inequality is valid.

A (necessarily bounded) domain $\Omega$ satisfies a quasihyperbolic boundary condition (more briefly, $\Omega$ is QHBC ) with respect to $x_{0} \in \Omega$ if there exists a constant $C \geq 1$ such that $k_{\Omega}\left(x, x_{0}\right) \leq C \log (C / \delta(x))$ for all $x \in \Omega$. We call $x_{0}$ the QHBC center of $\Omega$, and refer to the quasihyperbolic geodesic for $x, x_{0}$ as the QHBC path for $x$. The QHBC constant of $\Omega$ (with respect to $x_{0}$ ) is the smallest $C$ for which the above inequality is valid. The John and QHBC conditions depend only weakly on $x_{0}$ : if the condition is true for some choice of $x_{0} \in \Omega$, it is true for all such choices (however the John/QHBC constant increases without bound as $x_{0}$ tends to $\partial \Omega$ ).

The class of QHBC domains contains the class of John domains, which in turn includes all bounded Lipschitz domains and certain fractal regions (e.g. the region inside the von Koch snowflake). For an example of a QHBC domain that is not John, take a sequence of cubes $Q_{k}$ of sidelength $3^{-k}(k \in \mathbb{N})$, and attach each $Q_{k}$ to the unit cube $Q_{0}$ via a cubical neck of sidelength $4^{-k}$. Since, for large $k$, the necks are much narrower than the sidelength of $Q_{k}, \Omega$ cannot be John; it 
is, however, easy to construct QHBC paths (uniform in $k$ ).

We next define the concept of a domain with a slice property. This property is a version of the slice property introduced in [3] (condition (c) has been slightly weakened); it permits the construction of important test functions (tailored to the geometry of the domain) for Trudinger-type inequalities.

Definition 2.3. Suppose $\Omega \subset \mathbb{R}^{n}$ is a domain with a distinguished point $x_{0}$ and $C_{0}>1$. We say $\Omega$ has the $C_{0}$-slice property with respect to $x_{0}$ if, for each $x \in \Omega$, there is a path $\gamma:[0,1] \rightarrow \Omega, \gamma(0)=x_{0}, \gamma(1)=x$, and a pairwise disjoint collection of open subsets $\left\{S_{i}\right\}_{i=0}^{j}, j \geq 0$, of $\Omega$ such that:

(a) $x_{0} \in S_{0}, x \in S_{j}$, and $x_{0}$ and $x$ are in different components of $\Omega \backslash \bar{S}_{i}$ for all $0<i<j$.

(b) If $F \Subset \Omega$ is a curve containing both $x$ and $x_{0}$, and $0<i<j$, then $\operatorname{diam}\left(S_{i}\right) \leq C_{0} \operatorname{len}\left(F \cap S_{i}\right)$.

(c) $\gamma([0,1]) \subset \bigcup_{i=0}^{j} \bar{S}_{i}$.

(d) There exists $x_{i} \in \gamma_{i} \equiv \gamma([0,1]) \cap S_{i}$ such that $x_{0}$ is as previously defined, $x_{j}=x$, and $B\left(x_{i}, 2 r_{i}\right) \subset S_{i}$, where $r_{i}=C_{0}^{-1} \operatorname{diam}\left(S_{i}\right)$. Additionally, $\delta(x) \geq C_{0}^{-1} \operatorname{diam}\left(S_{i}\right)$, for all $x \in \gamma_{i}, 0 \leq i \leq j$.

We say that $\Omega$ has a slice property if it has the $C_{0}$-slice property for some $C_{0}$.

Condition (b) says that $S_{i}$ is about as thick as its diameter. The slice property does not permit a domain to have "flat" tentacles (e.g. the product of an exterior cusp and an interval), and restricts the geometry of small floating pieces of the boundary. We refer to the above path $\gamma$ as the slice path for $x$ (with respect to $x_{0}$ ). Many domains (including all simply-connected planar domains) satisfy a slice condition, as indicated by the following lemma which is taken from $[3]$.

Theorem 2.4. If $f$ is a $K$-quasiconformal mapping from a uniform domain $G \subset \mathbb{R}^{n}$ onto $\Omega$, then $\Omega$ has the $C_{1}$-slice property with respect to all of its points, for some constant $C_{1}=C_{1}(K, G)$. Additionally, any John domain $\Omega \subset \mathbb{R}^{n}$ satisfies a $C_{2}$-slice property with respect to $x_{0} \in \Omega$, for some constant $C_{2}=C_{2}\left(\Omega, x_{0}\right)$.

In the above theorem, the dependence of $C_{1}$ on $G$ is in fact only a dependence on $n$ and the constant of uniformity of $G$, and the dependence of $C_{2}$ on $\Omega$ and $x_{0}$ is only a dependence on $n$ and the John constant of $\Omega$ with respect to $x_{0}$.

Finally in this section, we define the main technical restrictions on measures that we shall need later. A positive Borel measure $\mu$ is locally doubling on $\Omega$ if there exists a constant $C_{\mu}$ such that $\mu\left(2 Q^{\prime}\right) \leq C_{\mu} \mu\left(Q^{\prime}\right)$ whenever $Q^{\prime}$ is a cube such that $2 Q^{\prime} \subset 2 Q$ for some $Q \in \mathcal{W}(\Omega)$. It is easy to deduce by a chaining argument that, for every $K \Subset \Omega$, there exists $C_{\mu}^{\prime}=C_{\mu}^{\prime}(K)$ such that $\mu\left(2 Q^{\prime}\right) \leq C_{\mu}^{\prime} \mu\left(Q^{\prime}\right)$ whenever $Q^{\prime}$ is a cube and $2 Q^{\prime} \subset K$ (in fact, $C_{\mu}^{\prime}$ can be 
chosen to depend only on $C_{\mu}$ and the quasihyperbolic diameter of $K$ ). We say that a weight $w$ is locally doubling on $\Omega$ if $w(x) d x$ is locally doubling on $\Omega$.

If $\mu$ is a locally doubling measure on $\Omega$, then a weight $f \not \equiv 0$ lies in the (local) Muckenhoupt class $A_{1}(\Omega, \mu)$ if there exists a constant $C_{f}$ such that whenever $Q$ is a cube and $2 Q \subset \Omega$, we have $f_{Q} f d \mu \leq C_{f} \inf _{Q} f$. If $\mu$ is Lebesgue measure (the only case of interest to us, except in the next paragraph), we simply write $A_{1}(\Omega)$.

Dual to $A_{1}(\Omega)$ is the notion of the (local) reverse Hölder class $R H_{\infty}(\Omega)$. We say that a weight $f \not \equiv 0$ lies in the (local) reverse Hölder class $R H_{\infty}(\Omega)$ if there exists a constant $C_{f}$ such that whenever $Q$ is a cube and $2 Q \subset \Omega$, we have $\sup _{Q} f \leq C_{f} f_{Q} f$. The spaces $A_{1}(\Omega)$ and $R H_{\infty}(\Omega)$ are intimately connectedfor example, it is easy to verify that $f \in R H_{\infty}(\Omega)$ if and only if $1 / f \in A_{1}(\Omega, \mu)$, where $\mu=f d x$. Clearly $A_{1}(\Omega)$ and $R H_{\infty}(\Omega)$ weights are locally doubling on $\Omega$.

In order to prove weighted Poincaré-type inequalities, it is often assumed in the literature that one or both weights satisfy a global doubling condition for all balls in $\mathbb{R}^{n}$; however, as suggested by the results of [6] and [2], this condition can often be replaced by a "strong doubling" assumption (so-called because it is stronger than local doubling). A positive Borel measure $\mu$ is in the strong doubling class $D_{t}(\Omega)$, where $t>1 / 2$, if there exists a constant $C$ such that $\mu(2 B \cap \Omega) \leq C \mu(B \cap \Omega)$ whenever $B$ is a ball and $(1 / t) B \subset \Omega$. We write $v \in D_{t}(\Omega)$ if $v$ is a weight such that $v(x) d x \in D_{t}(\Omega)$. This allows us to consider some important measures that may not be globally doubling (e.g. $\delta^{s} d x$ lies in $D_{t}(\Omega)$ for all $s>0$, but is not always extendable to a global doubling measure $[2])$.

\section{Conditions For $s$-TRudinger IMBEDdings}

Let us begin with a pair of elementary, but noteworthy, observations. First, $s$-Trudinger inequalities are equivalent to growth estimates on the constants for an associated family of Poincaré-type inequalities. In fact, using Stirling's formula, the reader can easily verify that (1.1) is equivalent to the following family of inequalities:

$$
\begin{aligned}
\exists C_{0}<\infty: \quad \text { for all } t & \geq 1, \text { for all } u \in \operatorname{Lip}(\Omega), \\
\left\|u-u_{\Omega, \nu}\right\|_{L^{t}(\Omega, \nu)} & \leq C_{0} t^{1 /(n+s)^{\prime}}\left\|\left|\nabla^{+} u\right|\right\|_{L^{n+s}(\Omega, \mu)}
\end{aligned}
$$

Secondly, $(\Omega, \nu, \mu)$ supports an $s$-Trudinger inequality if and only if

$$
\begin{aligned}
& \exists C_{0}<\infty: \quad \text { for all } u \in \operatorname{Lip}(\Omega), \\
& \quad\left\|u-u_{Q_{0}, \nu}\right\|_{\varphi_{s}(L)(\Omega, \nu)} \leq C_{0}\left\|\left|\nabla^{+} u\right|\right\|_{L^{n+s}(\Omega, \mu)},
\end{aligned}
$$

where $Q_{0} \in \mathcal{W}(\Omega)$ is fixed. To justify this, we may assume without loss of generality that $\nu(\Omega)<\infty$ (this fact is necessary for (1.1) to make sense, and, 
as we shall see, follows quite easily from (3.1)). The equivalence follows easily since the constant $u_{Q_{0}, \nu}-u_{\Omega, \nu}$ can be dominated by an $L^{1}(\Omega, \nu)$ norm of either $u-u_{\Omega, \nu}$ or $u-u_{Q_{0}, \nu}$ which in turn is less than the left-hand side of either (1.1) or (3.1). More generally, the above argument actually shows that $u_{\Omega, \nu}$ can be replaced by $u_{S, \nu}$ for any $S \subset \Omega, \nu(S)>0$ (with an imbedding constant that depends also on $\nu(S))$.

Given $s \geq 0$, we say that the measures $\nu, \mu$ are globally $s$-balanced if

$$
\begin{aligned}
& \exists c>0: \quad \text { for all } Q \in \mathcal{W}(\Omega) \\
& \quad \nu(Q) \leq c^{-1} \exp \left[-c \sum_{i=0}^{k}\left(\frac{\delta_{Q_{i}}^{n+s}}{\mu\left(Q_{i}\right)}\right)^{1 /(n+s-1)}\right]
\end{aligned}
$$

where $\left\{Q_{i}\right\}_{i=0}^{k}$ is the chain of Whitney cubes intersecting some particular path from a general point $x \in Q=Q_{k}$ to a fixed point $x_{0}$ in a fixed Whitney cube $Q_{0}$. The choices of $x_{0}$ and $Q_{0}$ are unimportant if $\nu, \mu$ are locally doubling, since the values of $\nu(Q), \mu(Q)$, and $\delta_{Q}$ vary by no more than some fixed factor as we let $Q$ vary over a set of Whitney cubes whose closures share a common point, and so the left-hand side of (3.2) changes by only a bounded amount when we make a new choice of $x_{0}$.

The global $s$-balance condition depends crucially on the geometry of the domain as well as on the weights. It generalizes the QHBC condition since, if $\nu$ is Lebesue measure and $d \mu=\delta^{s}(x) d x$, then $\nu, \mu$ are $s$-balanced if and only if $\Omega$ is QHBC. For general measures, however, the conditions can be rather different. For example, the domain in Example 5.5 is not QHBC and yet the weights are globally $s$-balanced. Moreover, there is no reason in general to expect that the QHBC path in a QHBC domain can be taken as the global $s$-balance path for a pair of globally $s$-balanced weights.

We say that $\nu, \mu$ are locally s-balanced if we merely have

(3.3) $\exists c>0$ : for all $Q \in \mathcal{W}(\Omega), \quad \nu(Q) \leq c^{-1} \exp \left[-c\left(\frac{\delta_{Q}^{n+s}}{\mu(Q)}\right)^{1 /(n+s-1)}\right]$.

If $d \nu=v d x, d \mu=w d x$, we say that $v, w$ are locally/globally $s$-balanced if $\nu, \mu$ are.

If $(\Omega, \nu, \mu)$ supports a 0 -Trudinger inequality, and $\nu, \mu$ satisfy certain technical conditions (including the fact that $d \mu=w d x$ ), we shall see that $w$ is bounded away from zero, and $\nu(\Omega)<\infty$ (this latter is also true for $s>0$ ). Under these assumptions on $\nu, \mu$, the local 0 -balance condition is trivially satisfied and the global 0 -balance condition simply says that there exists a constant $c>0$ such that for every Whitney cube $Q$ with center $x_{Q}$, we have 
$\nu(Q) \leq \exp \left(-c k_{\Omega}\left(x_{Q}, x_{0}\right)\right) / c$. In a QHBC domain, this is equivalent to the existence of constants $C, \varepsilon>0$ such that $\nu(Q) \leq C \delta_{Q}^{\varepsilon}$.

Let us now state two theorems involving $s$-Trudinger inequalities. The first establishes that the conditions mentioned above are often necessary, while the second shows that, together with an auxiliary condition (3.6), they are often sufficient.

Theorem 3.4. Suppose that $(\Omega, \nu, \mu)$ supports an $s$-Trudinger inequality, where $\Omega$ is a bounded domain, $\nu, \mu$ are locally doubling on $\Omega$, and $s \geq 0$. Then $\nu(\Omega)<\infty$ and $\nu, \mu$ are locally s-balanced. Furthermore,

(a) if $s=0$, and $d \mu=w d x$ for some $w \in A_{1}(\Omega) \cap R H_{\infty}(\Omega)$, then $w$ is bounded away from 0 on $\Omega$, and

(b) if $\mu \in D_{C_{1}}(\Omega)$, and $\Omega$ satisfies the $C_{0}$-slice condition for some $C_{0}<C_{1}$, then $\nu, \mu$ are globally s-balanced.

Part (a) of the above theorem may be a little surprising, as nothing like this can be proved for Poincaré inequalities, as can be seen by rescaling. As mentioned earlier, Trudinger-type inequalities are equivalent to a family of Poincaré inequalities, but any such rescaling affects the exponents in these Poincaré inequalities in a non-uniform way. The essential reason for (a) being true is that $p=n$ is the one exponent for which one can find a function which equals 1 on a ball $B, 0$ on $\mathbb{R}^{n} \backslash 2 B$, and whose gradient has $L^{p}$-norm bounded by a constant independent of the radius of $B$.

Theorem 3.5. Suppose that $\nu, \mu$ are Borel measures on a bounded domain $\Omega$, that $\nu$ is locally doubling on $\Omega$, and that $d \mu=w d x$ for some $w \in A_{1}(\Omega)$. In the case $s=0$, we additionally assume that $w$ is bounded away from zero, and that $d \nu=v d x$ for some $v \in R H_{\infty}(\Omega)$. If $\nu, \mu$ are globally s-balanced, and

$$
\exists \varepsilon>0: \quad \sum_{Q \in \mathcal{W}(\Omega)}[\nu(Q)]^{1-\varepsilon}<\infty
$$

then $(\Omega, \nu, \mu)$ supports an s-Trudinger inequality.

Since $\nu$ is locally doubling, the value of $\nu(Q)$ can change by no more than a bounded factor from one Whitney cube to an adjacent one. Thus (3.6) is true if

$$
\exists c>0: \quad \text { for all } \in \Omega, \quad \int_{\Omega} \exp \left(c k_{\Omega}\left(x, x_{0}\right)\right) d \nu(x)<\infty
$$

where $x_{0} \in Q_{0}$. If $\Omega$ is $\mathrm{QHBC}$, then it is easy to show that (3.7) is equivalent to

$$
\exists t>0: \quad \int_{\Omega} \delta^{-t} d \nu<\infty
$$


The conclusion of Theorem 3.5 may be false if we drop (3.6); see Theorem 5.6. Note also that if $\mu(Q) \leq \delta^{n+s}$ then the global $s$-balance condition clearly implies the following local version of (3.7):

$$
\text { for all } Q \in \mathcal{W}(\Omega): \quad \int_{Q} \exp \left(c k_{\Omega}\left(x, x_{0}\right)\right) d \nu(x)<\infty
$$

Condition (3.6) is a very weak restriction if $\Omega$ is QHBC. It was proved in this setting for Lebesgue measure by Smith and Stegenga [30]; in the next section we show that it is true for strong doubling measures. Consequently, we have the following theorem which says that the $s$-balance condition is in many cases necessary and sufficient for an $s$-Trudinger imbedding.

Theorem 3.10. Suppose that $s \geq 0$, and that $\Omega$ is a QHBC domain (with $\mathrm{QHBC}$ constant $\left.C_{\Omega}\right)$ satisfying the $C_{0}$-slice condition. Let $w \in A_{1}(\Omega) \cap D_{C_{1}}(\Omega)$ for some $C_{1}>C_{0}$, and suppose that $v \in R H_{\infty}(\Omega) \cap D_{C_{2}}(\Omega)$ for some sufficiently large constant $C_{2}$ depending only on $C_{\Omega}$. Then $(\Omega, v, w)$ supports an s-Trudinger inequality if and only if $v, w$ are globally $s$-balanced and, if $s=0, w$ is bounded away from zero.

\section{Proofs of imbedding theorems}

Proof of Theorem 3.4. We may assume without loss of generality that $\operatorname{diam}\left(Q_{0}\right)=1$. Let $u$ be any $C^{1}$ function which equals 0 on $Q_{0}, 1$ on $\Omega \backslash 2 Q_{0}$, and whose gradient is bounded. Clearly the right-hand side of (3.1) is finite and, if the left-hand side is finite, we must have $\nu\left(\Omega \backslash Q_{0}\right)<\infty$, and so $\nu(\Omega)<\infty$. For the rest of this proof, we normalize $\nu$ so that $\nu(\Omega)=1 / 2$.

Next, we fix an arbitrary $Q \in \mathcal{W}(\Omega), 2 Q \cap Q_{0}=\varnothing$. It is easy to construct a $C^{1}$ function $u: \Omega \rightarrow[0,1]$ which equals 1 on $Q, 0$ on $\Omega \backslash 2 Q$, and whose gradient is less than some fixed multiple of $\delta_{Q}^{-1}$. By elementary estimates and the $s$-Trudinger inequality, we conclude that

$$
\begin{aligned}
\left\|u-u_{Q_{0}}\right\|_{\varphi_{s}(L)(\Omega, \nu)} & \approx \log ^{-1 /(n+s)^{\prime}}\left(1+\nu(Q)^{-1}\right) \approx\left(\log \left[\nu(Q)^{-1}\right]\right)^{-1 /(n+s)^{\prime}} \\
& \lesssim\|\nabla u\|_{L^{n+s}(\Omega, \mu)} \approx\left(\frac{\mu(Q)}{\delta_{Q}^{n+s}}\right)^{1 /(n+s)}
\end{aligned}
$$

It follows easily that $\nu, \mu$ are locally $s$-balanced.

We next prove that $w$ is bounded below when $s=0$. Since $w \in A_{1}(\Omega) \cap$ $R H_{\infty}(\Omega)$, this is equivalent to showing that $w_{Q} \geq c$ for all $Q \in \mathcal{W}(\Omega)$, so let us fix an arbitrary $Q \in \mathcal{W}(\Omega), Q \neq Q_{0}$, and let $z$ be the center of $Q$. We define a Lipschitz function $u(x)=f(|x-z|)$, where $f(t)$ is zero for $t>\delta_{Q} / 2$, constant when $t \leq 2^{-\ell-1} \delta_{Q}$ (for a fixed but arbitrary $\ell \in \mathbb{N}$ ), and linear on each interval 
$I_{j} \equiv\left[2^{-j-1} \delta_{Q}, 2^{-j} \delta_{Q}\right], 1 \leq j \leq \ell$. We choose the slopes of $f$ on each of these intervals to be negative. Since we want $\|\nabla u\|_{L^{n}(\Omega, \mu)}=1$, we choose $f$ so that the $L^{n}(\mu)$-norm of $\nabla u$ on each of the associated annular regions is $\ell^{-1 / n}$. Using the fact that $w(x) \approx w_{Q}$ for all $x \in Q$, it follows that $\left|f^{\prime}(t)\right| \approx 2^{j} w_{Q}^{-1 / n} \ell^{-1 / n}$ on $I_{j}$ and that $f\left(2^{-j-1} \delta_{Q}\right)-f\left(2^{-j} \delta_{Q}\right) \approx w_{Q}^{-1 / n} \ell^{-1 / n}$. Thus, $u \approx w_{Q}^{-1 / n} \ell^{(n-1) / n}$ on $B=B\left(z, 2^{-\ell-1} \delta_{Q}\right)$. Applying the 0 -Trudinger inequality, we see that there exists $t_{0}>0$, not depending on $\ell$, such that

$$
\begin{aligned}
1 & \geq \int_{\Omega} \varphi_{0}\left(\frac{|u(x)|}{t_{0}}\right) d \nu(x) \geq \int_{B} \varphi_{0}\left(\frac{w_{Q}^{-1 / n} \ell^{(n-1) / n}}{C t_{0}}\right) d \nu(x) \\
& \gtrsim C_{\nu}^{-\ell-1} \nu(Q) \exp \left(\frac{c w_{Q}^{-1 /(n-1)} \ell}{C t_{0}{ }^{n /(n-1)}}\right),
\end{aligned}
$$

where $C_{\nu}$ is the local doubling constant of $\nu$. Taking logarithms and letting $\ell$ tend to infinity, we could then conclude that $w_{Q} \geq\left(C t_{0}^{n /(n-1)} \log C_{\nu}\right)^{-n+1}$.

Next supposing that $\Omega$ satisfies the $C_{0}$-slice condition and that $\mu \in D_{C_{0}}(\Omega)$, we wish to show that $\nu, \mu$ are globally $s$-balanced. Let $Q \in \mathcal{W}(\Omega)$ be arbitrary but fixed. The global $s$-balance condition is trivially true if $Q$ is close to $Q_{0}$ in the quasihyperbolic metric, so we may assume that $Q$ is sufficiently far from $Q_{0}$ to guarantee that $j>1$, where $\left\{S_{i}\right\}_{i=0}^{j}$ is the associated chain of slices for some fixed point $x \in Q$ (with respect to the center point $x_{0}$ ). By Definition 2.3(d), each slice $S_{i}, 0<i<j$, contains $2 B_{i}$ where $B_{i}=B\left(x_{i}, r_{i}\right)$. Writing $B=B\left(x, \delta(x) / C_{0}\right)$, we claim that (3.2) follows from the inequality

$$
\nu(B) \cdot \exp \left[c \sum_{i=1}^{j-1}\left(\frac{r_{i}^{n+s}}{\mu\left(B_{i}\right)}\right)^{1 /(n+s-1)}\right] \lesssim 1
$$

First we use doubling to get $\nu(B) \approx \nu(Q)$ and $r_{i}^{n+s} / \mu\left(B_{i}\right) \approx \delta_{Q_{i}}^{n+s} / \mu\left(Q_{i}\right)$, where $Q_{i}$ is the Whitney cube containing $x_{i}$. These cubes $Q_{i}$ do not completely cover the slice path. However, parts (c), (d) of the slice property ensure that we can fill the gaps by adding only a bounded number of extra cubes per gap (and each associated new summand is comparable in size to the summand associated with the closest point $x_{i}$ ).

Let us therefore prove (4.1). The definition of $r_{i}$ ensures that $C_{1} B_{i} \supset \bar{S}_{i}$ and so, by strong doubling, $\mu\left(B_{i}\right) \approx \mu\left(\bar{S}_{i} \cap \Omega\right)$. We define

$$
u_{i}(y)=a_{i} \mu\left(B_{i}\right)^{-1 /(n+s)}\left[\inf _{A \in \mathcal{F}_{y, x_{0}}} \operatorname{len}\left(A \cap S_{i}\right)\right], \quad y \in \Omega,
$$

where $\mathcal{F}_{y, x_{0}}$ is the set of rectifiable curves in $\Omega$ containing $y$ and $x_{0}$, len denotes 
arclength, and $a_{i}>0$ will be specified later. It is clear that $u_{i}$ is Lipschitz and, since the gradient of the bracketed factor is bounded in absolute value by 1 , we have

$$
\int_{\bar{S}_{i} \cap \Omega}\left|\nabla u_{i}\right|^{n+s} d \mu \lesssim a_{i}^{n+s}, \quad 0<i<j .
$$

On the other hand, Definition 2.3(b) tells us that

$$
u_{i}(y) \gtrsim r_{i} a_{i}\left(\mu\left(B_{i}\right)\right)^{-1 /(n+s)}=a_{i}\left(\frac{r_{i}^{n+s}}{\mu\left(B_{i}\right)}\right)^{1 /(n+s)}, \quad y \in Q .
$$

Letting $u=\sum_{i=1}^{j-1} u_{i}$, we have $u \equiv 0$ on $B_{0} \equiv B\left(x_{0}, \delta\left(x_{0}\right) / C_{0}\right)$, while

$$
u(y) \gtrsim \sum_{i=1}^{j-1} a_{i}\left(\frac{r_{i}^{n+s}}{\mu\left(B_{i}\right)}\right)^{1 /(n+s)}, \quad y \in B
$$

We have $\int_{\Omega}|\nabla u|^{n+s} d \mu \lesssim 1$, for any choice of $a_{i}$ satisfying $\sum_{i=1}^{j-1} a_{i}^{n+s} \approx 1$. To make such a choice while also maximizing the right-hand side of (4.2), we choose

$$
a_{i} \approx\left(\frac{r_{i}^{n+s}}{\mu\left(B_{i}\right)}\right)^{1 /(n+s)(n+s-1)}\left[\sum_{i=1}^{j-1}\left(\frac{r_{i}^{n+s}}{\mu\left(B_{i}\right)}\right)^{1 /(n+s-1)}\right]^{-1 /(n+s)}
$$

This choice of $a_{i}$ inserted in (4.2) gives us

$$
u(y) \gtrsim\left[\sum_{i=1}^{j-1}\left(\frac{r_{i}^{n+s}}{\mu\left(B_{i}\right)}\right)^{1 /(n+s-1)}\right]^{(n+s-1) /(n+s)}, \quad y \in B
$$

and so

$$
\int_{\Omega} \varphi_{s}\left(\frac{\left|u-u_{B_{0}, \nu}\right|}{t}\right) d \nu \geq \nu(B) \exp \left(\frac{1}{C t} \sum_{i=1}^{j-1}\left(\frac{r_{i}^{n+s}}{\mu\left(B_{i}\right)}\right)^{1 /(n+s-1)}\right) .
$$

By the $s$-Trudinger inequality, the left-hand side is bounded, so we are done.

Note that in the above proof we used the $s$-Trudinger inequality for Lipschitz functions, rather than just $C^{1}$ functions in two places, namely in the proofs of (a) and (b). In the first case, the test functions can easily be smoothened, but not in the second (at least with our very general slices). 
Proof of Theorem 3.5. We assume without loss of generality that $\nu(\Omega)=$ $1 / 2$ and $\operatorname{diam}\left(Q_{0}\right)=1$, where $Q_{0}$ is the central Whitney cube. Let $u \in \operatorname{Lip}(\Omega)$ be normalized so that $\|\nabla u\|_{L^{n+s}(\Omega, \mu)}=1$, but otherwise arbitrary, and let $k$ be the least integer greater than $n+s-1$; note that $\left|\nabla^{+} u\right|=|\nabla u| \mu$-almost everywhere because $d \mu=w d x$. Writing $\varphi_{s}^{0}(t)=\sum_{j=k}^{\infty} t^{j(n+s)^{\prime}} / j$ !, it suffices by Hölder's inequality to find a number $t \lesssim 1$ such that

$$
\int_{\Omega} \varphi_{s}^{0}\left(\frac{\left|u(x)-u_{Q_{0}, \nu}\right|}{t}\right) d \nu(x) \equiv \sum_{Q \in \mathcal{W}(\Omega)} \int_{Q} \varphi_{s}^{0}\left(\frac{\left|u(x)-u_{Q_{0}, \nu}\right|}{t}\right) d \nu(x) \lesssim 1 .
$$

As is often the case with Poincaré or Trudinger inequalities on general domains, we separately control "good" and "bad" terms, where the former are the terms in this last sum with $u_{Q_{0}, \nu}$ replaced by local constants $u_{Q, \nu}$, and the latter are the differences between the local and global constants. First we control the good terms:

$$
\begin{aligned}
\mathcal{G} & \equiv \sum_{Q \in \mathcal{W}(\Omega)} \int_{Q} \varphi_{s}^{0}\left(\frac{\left|u(x)-u_{Q, \nu}\right|}{t}\right) d \nu(x) \\
& =\sum_{j=k}^{\infty} \frac{t^{-j(n+s)^{\prime}}}{j !} \sum_{Q \in \mathcal{W}(\Omega)} \int_{Q}\left|u-u_{Q, \nu}\right|^{j(n+s)^{\prime}} d \nu .
\end{aligned}
$$

Let $Q \in \mathcal{W}(\Omega)$ be arbitrary. We first assume that $s=0$. By the classical Sobolev-Poincaré inequality, Hölder's inequality, and the fact that $v \in R H_{\infty}(\Omega)$, there exists $C_{1}=C_{1}(\nu)$ and $C_{2}=C_{2}(p, q, n)$ such that

$$
f_{Q}\left|u-u_{Q, \nu}\right|^{q} d \nu \leq C_{1} f_{Q}\left|u-u_{Q}\right|^{q} \leq C_{1} C_{2} \delta_{Q}^{q}\left(f_{Q}|\nabla u|^{p}\right)^{q / p}
$$

whenever $1 \leq p<\infty$ and $0<q \leq n p /(n-p)$ (or $q<\infty$ if $p \geq n$ ); see, for example, Chapter 7 of [17]. This is not quite good enough for us-we also need that if $q=j n^{\prime}, p \geq n$, then $C_{2}(p, q, n) \leq j ! a^{j}$, for some number $a=a(n)$, a fact which readily follows from the classical Trudinger inequality. Bearing in mind that $w \in A_{1}(\Omega)$, we deduce from this sharper version of (4.3) that

$$
\int_{Q}\left|u-u_{Q, \nu}\right|^{j n^{\prime}} d \nu \lesssim j ! a^{j}\left(\frac{\delta_{Q}^{n}}{\mu(Q)} \int_{Q}|\nabla u|^{n} d \mu\right)^{j /(n-1)} \nu(Q) .
$$


Since $w_{Q}$ is bounded away from zero, and $\int_{Q}|\nabla u|^{n} d \mu \leq 1$, we deduce that

$$
\mathcal{G} \lesssim \sum_{j=k}^{\infty} t^{-j n^{\prime}} a^{j} \sum_{Q \in \mathcal{W}(\Omega)} \nu(Q)
$$

Since $\nu(\Omega)<\infty, \mathcal{G}$ has been controlled for any choice of $t>a^{1 / n^{\prime}}$.

In the case $s>0$, we cannot afford to lose the $j$ ! factor. We use Lemma 2.1 to deduce

$$
f_{Q}\left|u-u_{Q}\right|^{q} d \nu \leq a^{q} \delta_{Q}^{q}\left(f_{Q}|\nabla u|^{p}\right)^{q / p}
$$

for some number $a$ dependent only on $n$. Thus

$$
\int_{Q}\left|u-u_{Q, \nu}\right|^{j(n+s)^{\prime}} d \nu \leq a^{j(n+s)^{\prime}}\left(\frac{\delta_{Q}^{n+s}}{\mu(Q)} \int_{Q}|\nabla u|^{n+s} d \mu\right)^{j /(n+s-1)} \nu(Q) .
$$

Writing $t_{1}=t^{1 / 2}$ and using the local $s$-balance condition, we get

$$
\frac{1}{j !} \cdot\left(\frac{a^{n+s} \delta_{Q}^{n+s}}{t_{1}^{n+s} \mu(Q)}\right)^{j /(n+s-1)} \leq \exp \left(c\left(\frac{\delta_{Q}^{n+s}}{\mu(Q)}\right)^{1 /(n+s-1)}\right) \lesssim \nu(Q)^{-1}
$$

provided that $t_{1} \gtrsim a$. Since $a$ depends only on $n$, it follows that for some $t \lesssim 1$,

$$
\mathcal{G} \lesssim \sum_{j=k}^{\infty} t_{1}^{-j(n+s)^{\prime}} \sum_{Q}\|\nabla u\|_{L^{n+s}(Q, \mu)}^{j(n+s)^{\prime}}
$$

Since $j>n+s-1$, the inner sum is less than $\|\nabla u\|_{L^{n+s}(\Omega, \mu)}^{j(n+s)^{\prime}}=1$. Thus $\mathcal{G}$ is controlled for some fixed $t_{1}=t^{1 / 2} \approx 1$.

We are left with controlling the bad terms

$$
\mathcal{B} \equiv \sum_{Q \in \mathcal{W}(\Omega)} \sum_{j=k}^{\infty} \frac{t^{-j(n+s)^{\prime}}}{j !}\left|u_{Q, \nu}-u_{Q_{0}, \nu}\right|^{j(n+s)^{\prime}} \nu(Q)
$$

By the triangle inequality, $\left|u_{Q, \nu}-u_{Q_{0}, \nu}\right| \leq \sum_{i=1}^{\ell}\left|u_{Q_{i}, \nu}-u_{Q_{i-1}, \nu}\right|$, where $\left\{Q_{i}\right\}_{i=0}^{\ell}$ denotes the chain of Whitney cubes in (3.2) in their natural order; of course, $\ell$ and the cubes $Q_{i}$ depend on $Q$. The definition of Whitney cubes ensures that the 
nine-fold dilate of any Whitney cube $P$ contains all Whitney cubes that share a boundary point with $P$, and so

$$
\left|u_{Q_{i}, \nu}-u_{Q_{i-1}, \nu}\right| \lesssim f_{9 Q_{i}, \nu}\left|u-u_{Q_{i-1}, \nu}\right| d \nu \lesssim f_{9 Q_{i}, \nu}\left|u-u_{9 Q_{i}, \nu}\right| d \nu
$$

Taking $q=1$ and $p=n+s$ in either (4.3) or (4.4) (depending on whether or not $s=0)$, we get

$$
\begin{aligned}
\mathcal{B} \lesssim & \sum_{j=k}^{\infty} \frac{t^{-j(n+s)^{\prime}}}{j !} \\
& \cdot \sum_{Q \in \mathcal{W}(\Omega)} \nu(Q)\left[\sum_{i=0}^{\ell}\left(\int_{9 Q_{i}}|\nabla u|^{n+s} d \mu\right)^{1 /(n+s)}\left(\frac{\delta_{Q_{i}}^{n+s}}{\mu\left(Q_{i}\right)}\right)^{1 /(n+s)}\right]^{j(n+s)^{\prime}},
\end{aligned}
$$

Applying Hölder's inequality (with exponent $n+s$ ) and the global $s$-balance condition to the innermost sum $I$ above, we see that

$$
\begin{aligned}
I & \leq\left(\sum_{i=0}^{\ell} \int_{9 Q_{i}}|\nabla u|^{n+s} d \mu\right)^{1 /(n+s)}\left(\sum_{i=0}^{\ell}\left(\frac{\delta_{Q_{i}}^{n+s}}{\mu\left(Q_{i}\right)}\right)^{1 /(n+s-1)}\right)^{1 /(n+s)^{\prime}} \\
& \lesssim\|\nabla u\|_{L^{n+s}(\Omega, \mu)} \cdot\left[\log \left(\frac{1}{c \nu(Q)}\right)\right]^{1 /(n+s)^{\prime}}=\left[\log \left(\frac{1}{c \nu(Q)}\right)\right]^{1 /(n+s)^{\prime}} .
\end{aligned}
$$

Assuming that $t>(C / \varepsilon)^{1 /(n+s)^{\prime}}$, we deduce that

$$
\begin{aligned}
\mathcal{B} & \lesssim \sum_{j=k}^{\infty} \frac{t^{-j(n+s)^{\prime}}}{j !} \sum_{Q \in \mathcal{W}(\Omega)} \nu(Q) C^{j}\left[\log \left(\frac{1}{c \nu(Q)}\right)\right]^{j} \\
& =\sum_{Q \in \mathcal{W}(\Omega)} \nu(Q) \sum_{j=k}^{\infty} \frac{\left(C t^{-(n+s)^{\prime}}\left[\log \left(\frac{1}{c \nu(Q)}\right)\right]\right)^{j}}{j !} \\
& \lesssim \sum_{Q \in \mathcal{W}(\Omega)} \nu(Q)^{1-\varepsilon} \lesssim 1 .
\end{aligned}
$$

Proof of Theorem 1.2. It is elementary to prove that $\int_{\Omega} \delta^{-1} d x=\infty$. The value of $t_{0}$ depends on the growth of the number of Whitney cubes of $\Omega$ of sidelength at least $2^{-k}$, and so is controlled by $d \equiv \operatorname{dim}_{M} \partial \Omega$, the Minkowski dimension of $\partial \Omega$ (see [27], [28], [8]), which easily leads to the inequality $t_{0} \leq d-n$ 
(however, $t_{0}$ may equal -1 even if $d=n$ ). Since the boundary of a QHBC domain $\Omega$ has Minkowski dimension strictly less than $n$ (see [30], [25]), it follows that $t_{0} \in[-1,0)$ as claimed. The rest of the theorem, except for the sharpness of $\varphi_{s}$, follows from Theorems 3.4 and 3.5 and the comments after those theorems. The sharpness of $\varphi_{s}$ is a special case of Theorem 5.1 below.

Note that for a bounded Lipschitz domain, or more generally a domain with boundary of Minkowski dimension $n-1$, we have $t_{0}=-1$ in Theorem 1.2. On the other hand, there are $\mathrm{QHBC}$ domains whose boundary dimension is arbitrarily close to $n$ - for the planar case, see [24], while in higher dimensions, we can simply take the product of a planar QHBC domain with dimension close to 2 and an $(n-2)$-dimensional cube. Therefore $t_{0}$ can be arbitrarily close to 0 .

Let us write $\Omega_{r}=\{x \in \Omega: \delta(x)<r\}$, where $\Omega \subset \mathbb{R}^{n}$ is a QHBC domain with QHBC constant $C_{\Omega}$. As mentioned above, $\operatorname{dim}_{M}(\partial \Omega)=d$ for some $d=$ $d\left(C_{\Omega}\right)<n$. It follows that, given $0<\alpha<n-d$, there exists $C=C(\Omega, \alpha)$ such that $\left|\Omega_{r}\right| \leq C r^{\alpha}$ for all $r>0$. More generally, we have the following result taken from [2], which we shall use to prove Theorem 3.10.

Lemma 4.5. Suppose that $\Omega$ is $\mathrm{QHBC}, \nu \in D_{C_{2}}(\Omega)$, and the constants $C_{\Omega}, C_{\nu}$ are as above. If $C_{2}$ is larger than some sufficiently large constant (dependent only on $n$ and $C_{\Omega}$ ), then there exist $C, \alpha>0$ dependent only on $n, C_{\Omega}$, and $C_{\nu}$ such that

$$
\text { for all } r>0: \quad \nu\left(\Omega_{r}\right) \leq C\left(\frac{r}{\operatorname{diam}(\Omega)}\right)^{\alpha} \nu(\Omega) .
$$

Proof of Theorem 3.10. It suffices to show that (3.6) follows from the given hypotheses. Without loss of generality, we assume that $\operatorname{diam}(\Omega)=1$. Defining $L_{i}=\Omega_{2^{-i+1}} \backslash \Omega_{2^{-i}}$, we see that any Whitney cube $Q$ whose center $z_{Q}$ lies in $L_{i}$ has sidelength comparable to $2^{-i}$, and the whole cube lies in $\Omega_{2^{-i+2}}$. Thus the number of such Whitney cubes is at most comparable to $2^{\text {in }}$. By Lemma 4.5,

$$
\sum_{Q \in \mathcal{W}(\Omega): z_{Q} \in L_{i}} \nu(Q)^{1-\varepsilon} \lesssim 2^{i n \varepsilon}\left(\sum_{Q \in \mathcal{W}(\Omega): z_{Q} \in L_{i}} \nu(Q)\right)^{1-\varepsilon} \lesssim 2^{i(n \varepsilon-\alpha(1-\varepsilon))}
$$

Choosing $0<\varepsilon<\alpha /(n+\alpha)$, we immediately deduce (3.6).

\section{Sharpness OF IMBEDDing RESUlts}

The local s-balance condition implies that for Whitney cubes $Q, \mu(Q) / \delta_{Q}^{n+s}$ cannot decrease very fast as one tends to the boundary, at least when $\Omega$ is QHBC and $\nu$ is locally doubling. To prove that the Orlicz function in an $s$-Trudinger 
inequality is sharp, or that this inequality implies that $\Omega$ must be QHBC, we shall roughly need to assume that $\mu(Q) / \delta_{Q}^{n+s}$ is bounded near one or all boundary points. If, however, $d \mu / d x \geq \delta^{t}$ for some $t<s$, the $s$-Trudinger inequality is not the sharp type of imbedding - in this case, the gradient norm often dominates the $L^{\infty}$ norm of the function, and one may even get a Hölder-type imbedding.

With a view to justifying the assertions in the above paragraph, let us first introduce some terminology. Given $0 \leq \alpha \leq 1$ and a closed path $\gamma \subset \Omega$, we write $k_{\alpha}(\gamma)=k_{\alpha, \Omega}(\gamma)=\int_{\gamma} \delta(z)^{\alpha-1}|d z|$. We then define the metric $k_{\alpha}(x, y)$ to be the infimum of $k_{\alpha}(\gamma)$, as $\gamma$ ranges over all closed paths in $\Omega$ linking $x, y \in \Omega$; note that $k_{0}$ is the quasihyperbolic metric and $k_{1}$ is the Euclidean metric. We define $\operatorname{Car}(\alpha), 0<\alpha<1$, the class of weak $\alpha$-carrot domains, to consist of all $k_{\alpha}$-bounded (and hence bounded) domains; we also write $\operatorname{Car}(0)=\mathrm{QHBC}$. It is shown in [3] that $\operatorname{Car}(\alpha) \subset \operatorname{Car}\left(\alpha^{\prime}\right)$ if and only if $\alpha \leq \alpha^{\prime}$; in particular, QHBC $\subset \operatorname{Car}(\alpha)$ for all $\alpha$. The related class $\operatorname{Cig}(\alpha) \subset \operatorname{Car}(\alpha)$ of weak $\alpha$-cigar domains, consists of all bounded domains $\Omega$ such that for some constant $C$ and all $x, y \in \Omega$, we have $k_{\alpha}(x, y) \leq C|x-y|^{\alpha}$.

When $\nu$ and $\mu$ are both Lebesgue measure, much more than (3.1) is true, and for a larger class of domains than QHBC. In fact, if $\|\nabla u\|_{L^{n+s}(\Omega)}<\infty$, then Theorems 4.1 and 4.3 of [3] tell us that:

(i) If $\Omega \in \operatorname{Cig}(s /(n+s-1))$, then $u \in C^{0, s /(n+s)}(\bar{\Omega})$.

(ii) If we merely have $\Omega \in \operatorname{Car}(s /(n+s-1))$, then $u$ is bounded.

The reader is invited to modify the arguments in [3] to get the following weighted results for functions $u$ such that $\|\nabla u\|_{L^{n+s}\left(\Omega, \delta^{t} d x\right)}<\infty, 0<t<s$ :

(iii) If $\Omega \in \operatorname{Cig}((s-t) /(n+s-1))$, then $u \in C^{0,(s-t) /(n+s)}(\bar{\Omega})$.

(iv) If we merely have $\Omega \in \operatorname{Car}((s-t) /(n+s-1))$, then $u$ is bounded.

The above examples suggest that $d \mu$ should not be substantially larger than $\delta^{s} d x$, at least "near part of the boundary", in order for the $s$-Trudinger inequality to be in any way sharp. We now prove such a sharpness result.

Theorem 5.1. Let $s \geq 0, K, R>0$, and let $\nu, \mu$ be positive measures on the bounded domain $\Omega \subset \mathbb{R}^{n}$. Let $\left(x_{m}\right)$ be a sequence in $\Omega$, tending to $z \in \partial \Omega$, such that $\left|x_{m}-z\right|<2 \delta\left(x_{m}\right)$, and let $r_{m}=\delta\left(x_{m}\right) / 2, B_{m}=B\left(x_{m}, r_{m}\right)$. If $\mu(B(z, t) \cap \Omega) \leq K t^{n+s}$ for all $t>0$, and $\nu\left(B_{m}\right) \geq r_{m}^{R} / K$ for every $m>0$, then (3.1) becomes false if we replace $\varphi_{s}$ by any Orlicz function $\psi$ such that $\psi(c t) / \varphi_{s}(t) \rightarrow \infty,(t \rightarrow \infty)$ for every $c>0$.

Proof. Let $x_{0}$ be the center of a fixed Whitney cube $Q_{0}$ of $\Omega$. Without loss of generality, we normalize $\Omega$ such that $\delta\left(x_{0}\right)=1$, and we assume that $r_{m}<1 / 6$ for all $m$. Let us now fix $m$, and write $x=x_{m}, r=r_{m}, B=B_{m}$. We define $B_{0}=B\left(x_{0}, 1 / 2\right)$, and let $M$ be the smallest integer $i$ for which $B\left(x, 2^{i+2} r\right)$ contains $x_{0}$. We define the annular slices $A_{j}=B\left(x, 2^{j} r\right) \backslash B\left(x, 2^{j-1} r\right)$ for every 
positive integer $j \leq M$. It is easily seen that $M \geq 2$ and that

$$
\log _{2}\left(\delta(x)^{-1}-1\right)-1<M \leq \log _{2}\left(\frac{\operatorname{diam}(\Omega)}{\delta(x)}\right) .
$$

We assume that $m$ (and hence $M$ ) is so large that $\log _{2}(2 / \delta(x)) \in[M / 2,2 M]$.

We choose $u(y)=g(|y-x|)$, where $g(t)$ is a decreasing Lipschitz function which is zero when $t>2^{M} r$, constant when $t<r$, and linear on $\left[2^{j-1} r, 2^{j} r\right]$, $1 \leq j \leq M$. Fixing $g$ by the equations $g\left(2^{j-1} r\right)-g\left(2^{j} r\right)=M^{-1 /(n+s)}$, it follows that $\int_{A_{j}}|\nabla u|^{n+s} d \mu \lesssim 1 / N$ and so $\|\nabla u\|_{L^{n+s}(\Omega, \mu)} \lesssim 1$.

Since $u(x)=0$ on $B_{0}$ and $u(x)=M^{(n+s-1) /(n+s)}$ on $B(x, r)$, we have

$$
\int_{\Omega} \psi\left(\frac{\left|u-u_{B_{0}}\right|}{t}\right) d \nu \gtrsim r^{R} \psi\left(\frac{M^{(n+s-1) /(n+s)}}{t}\right)
$$

Choosing $C>\log \left(2^{2 R+1}\right)$, we see that $e^{C M}-1>2^{M} r^{-R}-1$. If $t>0$ is fixed but arbitrary and $M$ is sufficiently large, our assumption on $\psi$ therefore implies that

$$
\int_{\Omega} \psi\left(\frac{\left|u-u_{B_{0}, \nu}\right|}{t}\right) d \nu>r^{R} \varphi_{s}\left((C M)^{(n+s-1) /(n+s)}\right)>2^{M}-r^{R} .
$$

Letting $m \rightarrow \infty$ (and hence $\left.M, r^{-1} \rightarrow \infty\right)$, it follows that $\left\|u-u_{B_{0}, \nu}\right\|_{\psi(L)(\Omega, \nu)}$ must be larger than any pre-ordained value $t$. Since $\|\nabla u\|_{L^{n+s}(\Omega, \mu)} \lesssim 1$, this contradicts the $s$-Trudinger inequality.

For sharpness of the QHBC class, we shall need an appropriate $\mu$-volume upper bound near all boundary points. To see this, let us write $w(x)=\left(x_{1}+1\right)^{s}$, $s>0$, where $x=\left(x_{1}, x^{\prime}\right) \in \mathbb{R} \times \mathbb{R}^{n-1}$, and consider the domain

$$
\Omega=\left\{x \in \mathbb{R}^{n}:|x|<1\right\} \cup\left\{x \in \mathbb{R}^{n}: 0<x_{1}<2,\left|x^{\prime}\right|<\left(\frac{1-x_{1}}{2}\right)^{t}\right\}
$$

where $1<t<(n+s-1) /(n-1)$. $\Omega$ is not a QHBC domain because of its external cusp, but $(\Omega, 1, w)$ supports an $s$-Trudinger inequality. To see this, note first that $\Omega_{1} \equiv\left\{x \in \Omega: x_{1}<0\right\}$ is QHBC and so $\left(\Omega_{1}, 1, w\right)$ supports an $s$-Trudinger inequality. Additionally, $\Omega_{2}=\left\{x \in \Omega: x_{1}>-1 / 2\right\}$ is an $s /(n+s-1)$-carrot domain, and so $\left(\Omega_{2}, 1, w\right)$ supports an $s$-Trudinger inequality by (ii) above. The claim now follows in a routine fashion.

We must also suitably restrict our class of domains in order to prove that the QHBC class is sharp (even if $s=0$ and $\nu, \mu$ are Lebesgue measure; see [3]). To this end we now define weak slice properties; as the name suggests, domains 
with a slice property also possess a weak slice property (simply let $m=j-1$ below). Also note that the conditions below are true for a null set of slices whenever $k_{\Omega}\left(x, x_{0}\right) \leq C_{0}-1$.

Definition 5.2. Suppose that $\Omega \subset \mathbb{R}^{n}$ is a domain with a distinguished point $x_{0}$ and that $C_{0}>1$. We say that $\Omega$ has the weak $C_{0}$-slice property with respect to $x_{0}$ if, for each $x \in \Omega$, there exists $m=m_{x} \geq 0$, and a pairwise disjoint collection of open subsets $S_{i}=S_{i, x}, 1 \leq i \leq m$, such that for all such $i$ :

(a) $B\left(x_{0}, \delta\left(x_{0}\right) / C_{0}\right)$ and $B\left(x, \delta(x) / C_{0}\right)$ are contained in different components of $\Omega \backslash \bar{S}_{i}$.

(b) $\operatorname{diam}\left(S_{i}\right) \leq C_{0} \operatorname{len}\left(F \cap S_{i}\right)$ for any curve $F \Subset \Omega$ containing both $x$ and $x_{0}$.

(c) $1+m \geq\left(1+k_{\Omega}\left(x, x_{0}\right)\right) / C_{0}$.

$\Omega$ has a weak slice property if it has the weak $C_{0}$-slice property for some $C_{0}$.

In the above definition, we claim that $\delta(x) \lesssim \operatorname{diam}\left(S_{i}\right)$ for all $x \in S_{i}$, $1 \leq i \leq m$. If not, then $2 B$ is disjoint from $B\left(x_{0}, \delta\left(x_{0}\right) / 2 C_{0}\right) \cup B\left(x, \delta(x) / 2 C_{0}\right)$ for some ball $B=B(z, \delta(z) / 200) \supset S_{i}$. It is easy to see that any path from $x$ to $x_{0}$ that passes through $2 B$ can be deformed to avoid passing through $B$, contradicting Definition 5.2(b) and proving our claim.

QHBC domains do not necessarily have a slice property, but they always have a weak slice property. To construct an example of a QHBC domain $\Omega \subset \mathbb{R}^{3}$ which does not have a slice property, we glue together the following pieces: the unit cube $Q_{0}$, a sequence of cubes $Q_{k}$ of sidelength $3^{-k}$, and a sequence of rectangular boxes $R_{k}$ of dimensions $3^{-k} \times 4^{-k} \times 4^{-k}$ (for each $k \in \mathbb{N}$ ). For each $k$, we glue one of the four longer faces of each $R_{k}$ completely to a face of $Q_{k}$ and then glue the opposite face of $R_{k}$ completely to some face of $Q_{0}$ (all done without overlap, of course). It is easy to show that any such domain $\Omega$ is QHBC. However, since the length of $R_{k}$ is much greater than its width or depth, $\Omega$ cannot have a slice property (paths from the centers of $Q_{k}$ to the center of $Q_{0}$ cannot be nicely sliced).

To see that a QHBC domain $\Omega$ (with distinguished point $x_{0}$ ) has a weak slice property, we normalize $\Omega$ so that $\delta\left(x_{0}\right)=1$. The weak slice condition is easily seen to hold for points $x \in \Omega$ such that $\delta(x) \geq 1 / 8$, since then $k_{\Omega}\left(x, x_{0}\right)$ is bounded. If $\delta(x)<1 / 8$, we define the slices $S_{i}$ to be the annular regions $S_{i}=B\left(x, 2^{i} \delta(x)\right) \backslash B\left(x, 2^{i-1} \delta(x)\right)$, for $i=1, \ldots, m$, where $m=\left\lfloor\log _{2}(1 / d(x))-\right.$ $1\rfloor$. For any domain, these slices automatically satisfy conditions (a) and (b) of Definition 5.2, and condition (c) follows from the fact that $\Omega$ is QHBC.

We now state a QHBC-sharpness result for $s$-Trudinger inequalities. Together with Theorem 1.2, and the fact that QHBC domains have a weak slice property, this immediately gives an $s$-Trudinger characterization of QHBC domains as a corollary. 
Theorem 5.3. Let $s \geq 0$ and let $\Omega \subset \mathbb{R}^{n}$ be a bounded domain satisfying a weak slice property with respect to $x_{0} \in \Omega$. Let $\nu, \mu$ be positive measures on $\Omega$, and suppose that there exist $K, R>0$ such that $\mu(B(z, t) \cap \Omega) \leq K t^{n+s}$ and $\nu(Q) \geq \delta_{Q}^{R} / K$ for all $t>0, z \in \Omega$, and $Q \in \mathcal{W}(\Omega)$. If $(\Omega, \nu, \mu)$ supports an $s$-Trudinger inequality, then $\Omega$ is a QHBC domain.

Corollary 5.4. Let $s, t \geq 0$ and let $\Omega \subset \mathbb{R}^{n}$ be a bounded domain. Then $\Omega$ is QHBC if and only if it has a weak slice property and $\left(\Omega, \delta^{t}, \delta^{s}\right)$ supports an $s$-Trudinger inequality.

A weaker version of Theorem 5.3 follows easily from Theorem 3.4. If we assume that $\Omega$ has a slice property (rather than merely a weak slice property), and if the other hypotheses of Theorem 5.3 are satisfied, then the global $s$ balance condition implies that $\nu(Q) \leq c^{-1} \exp \left(-c k_{\Omega}\left(x, x_{0}\right)\right)$ for any $x \in Q$. Since $\nu(Q) \gtrsim \delta_{Q}^{R} \gtrsim \delta^{R}(x)$, it follows readily that $\Omega$ is QHBC.

Example 5.5. Some condition similar to $\nu(Q) \geq \delta_{Q}^{R}(x) / K$ is needed in Theorem 5.3. To see this, fix $t>1, s \geq 0, c>0$, and define

$$
\begin{aligned}
& \Omega=\left\{x \in \mathbb{R}^{n}:|x|<1\right\} \\
& \cup\left\{x=\left(x_{1}, x^{\prime}\right) \in \mathbb{R} \times \mathbb{R}^{n-1}: 0<x_{1}<2,\left|x^{\prime}\right|<\left(1-\frac{x_{1}}{2}\right)^{t}\right\} .
\end{aligned}
$$

Then $\Omega$ is not QHBC but (3.7), and hence also (3.6), is true for the locally doubling measure $d \nu=\exp \left(-c k_{\Omega}\left(x, x_{0}\right)\right) d x$. It is also clear that $\nu$ and $\mu$ are globally $s$-balanced if $d \mu=\delta^{s} d x$, and that the other conditions in Theorem 3.5 are fulfilled. We conclude that $(\Omega, \nu, \mu)$ supports an $s$-Trudinger inequality. It is not difficult to see that $k_{\Omega}\left(x, x_{0}\right) \leq C \delta(x)^{1 / t-1}$, and so the assumption $\nu(Q) \geq \delta_{Q}^{R}(x) / K$ is false, but "not by much" if $t$ is near 1 .

Proof of Theorem 5.3. Let $\left\{S_{i}\right\}_{i=1}^{m}$ be the weak $C_{0}$-slices associated with $x \in \Omega$. Without loss of generality, we may assume $C_{0}>2$ and $m \geq 2$ (since the QHBC condition is trivially satisfied for $k_{\Omega}\left(x, x_{0}\right)<3 C_{0}$, and $m+1 \gtrsim$ $\left.\left(1+k_{\Omega}\left(x, x_{0}\right)\right) / C_{0}\right)$.

Writing $\delta_{i}=\operatorname{diam}\left(S_{i}\right)$ and $\mathcal{F}_{y, x_{0}}$ for the set of all rectifiable curves in $\Omega$ containing $y$ and $x_{0}$, we define $u=\sum_{i=1}^{m} u_{i}$, where

$$
u_{i}(y)=\frac{m^{-1 /(n+s)}}{\delta_{i}}\left[\inf _{A \in \mathcal{F}_{y, x_{0}}} \operatorname{len}\left(A \cap S_{i}\right)\right], \quad y \in \Omega .
$$

It is clear that $u_{i}$ is Lipschitz and, since $\delta(x) \lesssim \delta_{i}$ for all $x \in S_{i}$, we have $\int_{\Omega}\left|\nabla u_{i}\right|^{n+s} d \mu \lesssim 1 / m$. It is also clear that $u_{i} \gtrsim m^{-1 /(n+s)}$ on $B \equiv B\left(x, \delta(x) / C_{0}\right)$. 
We deduce that $\|\nabla u\|_{L^{n+s}(\Omega, \mu)} \lesssim 1$ and $u \gtrsim m^{(n+s-1) /(n+s)}$ on $B$. Since $u=0$ on $B_{0} \equiv B\left(x_{0}, \delta\left(x_{0}\right) / C_{0}\right)$, we deduce that

$$
\int_{\Omega} \varphi_{s}\left(\frac{\left|u-u_{B_{0}, \nu}\right|}{t}\right) d \nu \geq \int_{B} \varphi_{s}\left(\frac{u(y)}{t}\right) d \nu \gtrsim \delta(x)^{R}\left[e^{m t^{-(n+s) /(n+s-1)}}-1\right]>1
$$

whenever $t^{(n+s) /(n+s-1)}<m / \log \left(1+\delta(x)^{-R}\right)$. Thus,

$$
\|u\|_{\varphi_{s}(L)(\Omega, \mu)} \geq\left[\frac{m}{\log \left(1+\delta(x)^{-R}\right)}\right]^{(n+s-1) /(n+s)} .
$$

The $s$-Trudinger inequality now implies that $k_{\Omega}\left(x, x_{0}\right) \lesssim \log \left(1+\delta(x)^{-R}\right)$, which is equivalent to the desired inequality.

We now show that the hypothesis (3.6) is essential in Theorem 3.5. Below, we write $h \in D(0, \infty)$ if $h:(0, \infty) \rightarrow(0, \infty)$ satisfies the doubling condition:

$\exists C \in(0, \infty): \quad$ for all $x, y, \quad 0<\frac{x}{2}<y<2 x \Rightarrow \frac{h(x)}{C}<h(y)<C h(x)$.

Theorem 5.6. Suppose that $\Omega$ is the unit ball in $\mathbb{R}^{n}, z \in \partial \Omega, s \geq 0$, and $C_{0}>1$. Let $\nu, \mu$ be positive measures on $\Omega$ such that $d \nu=v d x, d \mu=$ $w d x, v(x)=|x-z|^{-n} f(|x-z|), w(x)=|x-z|^{s} g(|x-z|)$, and $f, g \in D(0, \infty)$. Writing $a_{j}=f\left(2^{-j}\right), b_{j}=g\left(2^{-j}\right)$ for $j \in \mathbb{N}$, we additionally suppose that $\left(a_{j}\right) \in \ell^{1} \backslash \bigcup_{0<p<1} \ell^{p}$, and that:

$$
\text { for all } i \in \mathbb{N}: \quad C_{0} \exp \left(\sum_{j=1}^{i} b_{j}^{-1 /(n+s-1)}\right) \geq a_{i}^{-1 / C_{0}},
$$

$$
\text { for all } i>1: \quad C_{0} \sum_{j=1}^{\lfloor i / 2\rfloor} b_{j}^{-1 /(n+s-1)} \geq \sum_{j=1}^{i} b_{j}^{-1 /(n+s-1)} \text {. }
$$

Then $(\Omega, \nu, \mu)$ does not support an s-Trudinger inequality.

Some comments on the hypotheses in the above theorem are in order. The condition $\left(a_{j}\right) \in \ell^{1}$ is equivalent to $\nu(\Omega)<\infty$ and so it could be dropped as an assumption, since any $s$-Trudinger imbedding implies $\nu(\Omega)<\infty$ (Theorem 3.4). A little calculation shows that $\left(a_{j}\right) \notin \bigcup_{0<p<1} \ell^{p}$ if and only if $\nu$ fails to satisfy (3.6); it is also easy to see that the global $s$-balance condition for a point along the radius to $z$ is equivalent to an inequality in the opposite direction to (5.7). Note also that, since $\left(a_{j}\right) \in \ell^{1},(5.7)$ implies that $\sum_{j} b_{j}^{-1 /(n+s-1)}=\infty$. Thus, 
(5.8) simply says that this series diverges slowly. Finally, the hypothesis that $\Omega$ is a ball can be considerably relaxed-we use this only to get that $\left|S_{i}\right| \approx 2^{-i n}$, but this is true if, for example, $\Omega \cap B(z, r)$ is a John domain for some fixed $r>0$.

It is easy to check that the functions $f(t)=[\log (3 / t)]^{-1}[\log \log (10 / t)]^{-2}$, $g(t)=[\log (3 / t)]^{n+s-1}$ satisfy the conditions in the above theorem, and it is obvious that the resulting measures $\nu, \mu$ satisfy all except two of the hypotheses of Theorem 3.5. The exceptions are (3.6), which is clearly false, and the global $s$-balance condition which, although true, deserves some words of justification.

If $s>0$ and $0<r<1$, the values of $v$ and $1 / w$ at $r z$ are within a bounded factor of the maximum values of these functions on the spherical shell $\{|x|=r\}$, and so it suffices to verify the balance condition only for $x=r z, \frac{1}{2}<r<1$. Let $L$ be the line segment from 0 to $r z$, and let $\left(Q_{i}\right)_{i=1}^{j}$ be the sequence of Whitney cubes that intersect $L$. Since only a bounded number of these cubes intersect each subset $L \cap S_{k}$, we have

$$
\begin{aligned}
E & \equiv \exp \left(-c \sum_{j=1}^{i}\left(\frac{\delta_{Q_{j}}^{n+s}}{w\left(Q_{j}\right)}\right)^{1 /(n+s-1)}\right) \gtrsim \exp \left(-C c \sum_{k=1}^{\left\lfloor\log _{2}(1-r)\right\rfloor} k^{-1}\right) \\
& \gtrsim\left[\log _{2}(1-r)\right]^{-C c}
\end{aligned}
$$

where $C>0$ is fixed. If we choose $c<1 / C$, this is easily seen to dominate $\nu\left(Q_{j}\right)$.

In the case $s=0$, the above analysis still shows that the balance condition is valid along the line segment from 0 to $z$ but, since $1 / w(x)$ is no longer nearly maximal at $r z$, we must also consider other points. Any other line segment from 0 to $z^{\prime} \in \partial \Omega$ can be divided into two parts: an inner part $L_{1}=\left\{r z^{\prime}: 0 \leq r \leq\right.$ $\left.1-\left|z-z^{\prime}\right| / 4\right\}$, and an outer part $L_{2}=L \backslash L_{1}$. Since distance to $z$ and distance to $\partial \Omega$ are comparable on $L_{1}$, the previous analysis readily extends to show that the balance condition is satisfied on $L_{1}$. If $r z^{\prime} \in L_{2}$, then we separately estimate $E$ over those cubes $Q_{j}$ intersecting $L_{1}$ and $L_{2}$; on the latter set $\left|r z^{\prime}-z\right| \approx\left|z^{\prime}-z\right|$, and we readily get an absolute lower bound for this part of the exponential sum.

Proof of Theorem 5.6. Let $M$ be a fixed but arbitrary integer greater than 1 and, for all $i \in \mathbb{N}$, let $S_{i}=\left\{x \in \Omega: 2^{-i-1}<|x-z| \leq 2^{-i}\right\}, T_{i}=\bigcup_{j \geq i} S_{j}$. For $1 \leq i \leq M$, we define $u(y)=\sum_{i=1}^{M} u_{i}(y)$, where

$$
\begin{aligned}
u_{i}(y) & =2^{i} b_{i}^{-1 /(n+s)} c_{i}\left[\inf _{A \in \mathcal{F}_{y, 0}} \operatorname{len}\left(A \cap S_{i}\right)\right], \quad y \in \Omega \\
c_{i} & =\frac{b_{i}^{-1 /(n+s)(n+s-1)}}{\left(\sum_{j=1}^{M} b_{j}^{-1 /(n+s-1)}\right)^{1 /(n+s)}} .
\end{aligned}
$$


We then have $\left\|\nabla u_{i}\right\|_{L^{n+s}(\Omega, \mu)} \approx c_{i}$, and so $\|\nabla u\|_{L^{n+s}(\Omega, \mu)} \approx 1$. Also

$$
u(y) \geq \sum_{j=1}^{i-1} b_{j}^{-1 /(n+s)} c_{j}=\frac{\sum_{j=1}^{i-1} b_{j}^{-1 /(n+s-1)}}{\left(\sum_{j=1}^{M} b_{j}^{-1 /(n+s-1)}\right)^{1 /(n+s)}}, \quad y \in T_{i} .
$$

If $i>M / 2$, then (5.8) implies that

$$
u(y) \gtrsim\left(\sum_{j=1}^{i-1} b_{j}^{-1 /(n+s-1)}\right)^{(n+s-1) /(n+s)}, \quad y \in T_{i} .
$$

Now applying (5.7), we see that

$$
\int_{\Omega} \varphi_{s}\left(\frac{|u|}{t}\right) d \nu \geq \sum_{M / 2<i \leq M} \int_{S_{i}} \varphi_{s}\left(\frac{|u|}{t}\right) d \nu \gtrsim \sum_{M / 2<i \leq M} a_{i}^{1-1 / C C_{0} t},
$$

where $C$ is fixed. Thus $\|u\|_{\varphi_{s}(L)(\Omega, \nu)}$ cannot be uniformly bounded as $M \rightarrow \infty$. After the usual smoothening of $u$, the theorem follows.

\section{IMBEDDINGS IN NON-EUCLIDEAN DOMAINS}

Let $(X, d)$ be a metric space and suppose that there is a measure $\lambda$ on $X$ which is doubling at small scales, in the sense that there exist numbers $0<R \leq$ $\infty, 0<C_{d}<\infty$, such that $\lambda(2 B) \leq C_{d} \lambda(B)$, whenever $B$ is a metric ball of radius at most $R$. In this case we say that $(X, d, \lambda)$ is a homogeneous space (and $R, C_{d}$ will have the above meanings); for more details about such spaces, we refer the reader to the book of Coifman and Weiss [10]. A "length space" is a metric space in which distance between points is the infimum of the lengths of rectifiable curves joining those points. For brevity, we define an HL-space to be a homogeneous space which is also a length space. This section concerns generalizations of Theorem 3.5 to certain HL-spaces.

We write $B(x, r)$ for the (open) metric ball centered at $x$ with radius $0<$ $r<\infty$; as before, if $B=B(x, r)$, and $0<t<\infty$, we write $t B$ for its $t$-fold dilate $B(x, t r)$. Suppose $\Omega$ is a connected open proper subset of $X$ whose diameter is less than $2 R$, and let $\delta(x)=d(x, X \backslash \Omega)$ for every $x \in \Omega$. We define the weight classes $A_{1}(\Omega, \lambda)$ and $R H_{\infty}(\Omega, \lambda)$ in the obvious way ( $\lambda$-averages of $w$ over metric balls replacing Lebesgue averages of $w$ over Euclidean balls).

Instead of decomposing $\Omega$ into disjoint Whitney cubes, we decompose it into a countable union of balls of the form $B(x, \delta(x) / 20)$ whose (1/5)-dilates are disjoint and whose 5 -dilates have bounded overlap at any point of $\Omega$. Denoting by $\mathcal{W}(\Omega)$ any fixed collection of this type, we call it "the" Whitney covering of $\Omega$; we call any $B \in \mathcal{W}(\Omega)$ a Whitney ball and write $\delta_{B}$ for its radius. 
It is a rather routine argument to show that such Whitney coverings exist, but let us sketch the idea for completeness. First, a standard covering lemma (see e.g. [21, Theorem 13.12]) applied to the set of all balls $B(x, \delta(x) / 100)$, $x \in \Omega$, gives everything except for the bounded overlap of the dilated balls. If $B, B^{\prime} \in \mathcal{W}(\Omega)$ with $5 B$ and $5 B^{\prime}$ overlapping, then elementary estimation shows that their radii differ by a factor at most 3 , and so $(1 / 5) B^{\prime} \subset 21 B$. By the doubling property, $\mu\left((1 / 5) B^{\prime}\right) \gtrsim \mu(21 B)$, so disjointness of the $(1 / 5)$-dilates of Whitney balls bounds the number of balls $5 B^{\prime}, B^{\prime} \in \mathcal{W}(\Omega)$, that can intersect $5 B$. Note also that if in fact $B$ and $B^{\prime}$ intersect, their radii are so similar that the 5 -fold dilate of either ball contains the double of the other one.

Below, we use the term "global $s$-balance condition" to refer to the general version of (3.2) which is obtained by replacing Whitney cubes with those balls $B \in \mathcal{W}(\Omega)$ that intersect some path from $x$ to a fixed point $x_{0}$. In this balance condition and elsewhere, we also replace $n$ by a number $N>1$, which occurs in our general imbeddings below. The number $N$ is an abstract replacement for Euclidean dimension (in a Hörmander vector field context, $N$ is the homogeneous dimension, which is usually larger than the topological dimension).

$H L$-spaces do not have to support any imbeddings of Poincaré or Trudinger type. In order to prove $s$-Trudinger inequalities, we shall assume as axioms certain inequalities for balls; later we discuss various situations in which these axioms are satisfied. Our axioms say that, for a fixed pair of functions $f$ and $g$, there is a number $N>1$ and constants $C, C_{p}>0$ such that the following abstract $L^{\infty}$ and Trudinger inequalities are valid on all balls $B=B(x, r), r \leq R$. For all $y \in B, p>N$ :

$$
\begin{aligned}
|f(x)-f(y)| & \leq C_{p} r\|g\|_{L^{p}\left(2 B, \lambda_{2 B}\right)}, \\
\left\|f-f_{B, \lambda}\right\|_{\varphi(L)\left(B, \lambda_{B}\right)} & \leq C r\|g\|_{L^{N}\left(2 B, \lambda_{2 B}\right)},
\end{aligned}
$$

where $\varphi(x)=\exp \left(x^{N^{\prime}}\right)-1$, and $\lambda_{B}$ is the probability measure on $B$ given by $\lambda_{B}(E)=\lambda(E) / \lambda(B)$ (thus $\lambda_{B}$ and $\lambda_{2 B}$ are equivalent on $B$ ). No explicit relationship between $f$ and $g$ is assumed, but one should intuitively think of $g$ as a replacement for $|\nabla f|$.

Theorem 6.1. Let $(X, d, \lambda)$ be an $H L$-space, let $\Omega$ be a connected open set in $X$ of diameter less than $R$, and let $\nu, \mu$ be Borel measures on $\Omega$, where $\nu$ is locally doubling and $d \mu=w d \lambda$ for some $w \in A_{1}(\Omega, \lambda)$. If $s=0$, we additionally assume that $w$ is bounded away from zero and that $d \nu=v d x$ for some $v \in R H_{\infty}(\Omega, \lambda)$. If $f, g$ satisfy $\left(L^{\infty}\right)$ and $(T r), \nu, \mu$ are globally s-balanced, $\varphi_{s}(x)=\exp \left(x^{(N+s)^{\prime}}\right)-1$, and

$$
\exists \varepsilon>0: \quad \sum_{B \in \mathcal{W}(\Omega)} \nu(B)^{1-\varepsilon}<\infty
$$


then there exists a constant $C_{0}$ such that $\left\|f-f_{\Omega, \nu}\right\|_{\varphi_{s}(L)(\Omega, \nu)} \leq C_{0}\|g\|_{L^{N+s}(\Omega, \mu)}$. Furthermore, $C_{0}$ depends on the constants in $\left(L^{\infty}\right)$ and $(T r)$, but not otherwise on $f$ or $g$.

Sketch of proof. Let us merely point out how the proof of Theorem 3.5 needs to be modified. As before, we split into good and bad parts, where good terms have the form $\int_{B}\left|u-u_{B, \nu}\right|^{j(N+s)^{\prime}} d \nu$, for some $B \in \mathcal{W}(\Omega)$. In the case $s=0$, we argue as before, but using (Tr), to get

$$
\begin{aligned}
\int_{B}\left|u-u_{B, \nu}\right| N^{\prime} d \nu & \lesssim j ! a^{j} \delta_{B}^{j N^{\prime}}\left(f_{2 B} g^{N} d \lambda\right)^{j /(N-1)} \nu(B) \\
& \lesssim j ! b^{j}\left(\delta_{B}^{N} f_{2 B} g^{N} d \mu\right)^{j /(N-1)} \nu(B) \\
& \lesssim j ! c^{j}\left(\frac{\delta_{B}^{N}}{\mu(B)} \int_{2 B} g^{N} d \mu\right)^{j /(N-1)} \nu(B)
\end{aligned}
$$

where $a, b, c>0$ are independent of $j$. The case $s>0$ is different only in that we use $\left(L^{\infty}\right)$ in place of Lemma 2.1 ; we also use the fact that $w \in A_{1}(\Omega, \lambda)$ to replace $\mu(2 B)$ by the comparable $\mu(B)$ before using the local balance condition. In each case, we integrate powers of $g$ over double dilates of Whitney balls; these sum nicely by bounded overlap.

As for the bad terms, the modifications to the argument there are similar to those for the good terms with one extra minor change: since the 5 -fold dilate of any Whitney ball contains the double dilates of all overlapping Whitney balls, we only need to dilate the balls by a factor of 5 rather than the factor of 9 used in the proof of Theorem 3.5.

For Theorem 6.1 to be of genuine interest, we need to know a variety of situations in which (6.2), the global $s$-balance condition, $\left(L^{\infty}\right)$, and $(\operatorname{Tr})$ are valid. The proof that strong doubling implies (3.6) for Euclidean QHBC domains (contained in Lemma 4.5 and part of Theorem 3.10) extends without difficulty ${ }^{1}$ to prove that strong doubling implies (6.2) when $\Omega$ is a metric ball (or more generally a metric John domain; see [7] and [16] for more details on such domains) in an HL-space. It is also readily verified that the global $s$-balance condition is satisfied if $\Omega$ is a metric ball (or metric John domain), $d \mu=\delta^{s} d \lambda$, and $d \nu=\delta^{t} d \lambda$ for some $t \geq 0$.

We now examine situations in which $\left(L^{\infty}\right)$ and $(\operatorname{Tr})$ are valid. A large class of examples consists of the Carnot-Carathéodry spaces of Garofalo and Nhieu

\footnotetext{
${ }^{1}$ One part of the proof of Lemma 4.4 given in [2] depends on either the Besicovitch covering lemma or the "5-dilate" covering lemma that we used above. In general spaces we do not have the former, but the latter is of course valid.
} 
[16]. As usual with such spaces, the metric is defined in terms of sub-unit curves whose derivatives are almost everywhere linear combinations of certain Lipschitz continuous vector fields. The authors introduce hypotheses, labelled (H.1)-(H.3), which roughly say that the metric space is doubling at small scales, satisfies a "weak (1,1)-Poincaré inequality", and is a complete length space. Under the assumptions (H.1)-(H.3), inequalities $\left(L^{\infty}\right)$ and $(\operatorname{Tr})$ are also true (see Theorems 1.9 and 1.11 of [16]); furthermore it is assumed that $N=\log _{2} C_{d}$ (so $N$ equals the ambient dimension in the Euclidean context with Lebesgue measure).

Several examples of Carnot-Carathéodory spaces satisfying hypotheses (H.1) (H.3) are given in [16]. These examples include stratified nilpotent Lie groups and, more generally, connected Lie groups with polynomial volume growth, as well as spaces on which the vector fields are of Grushin or Hörmander type, and complete $n$-dimensional Riemannian manifolds with non-negative Ricci tensor. In each of these cases, $f$ can be any smooth function, and $g$ has a specific interpretation as the absolute value of some sort of horizontal or Riemannian gradient of $f$.

The work of Franchi, Lu, and Wheeden [15] and of Hajłasz and Koskela [21] leads to another large class of examples. Suppose that $(X, d, \lambda)$ is an HL-space and $B$ is a fixed ball in $X$ of radius $r \leq R / 2 . N$ is allowed to be any number so large that $\lambda\left(t B^{\prime}\right) \geq C_{N} t^{N} \lambda\left(B^{\prime}\right)$ for all balls $B \subset X$, all sub-balls $B^{\prime}$ of $2 B$, and all $0<t<1$ (with a uniform constant $C_{N}>0$ ). Note that this volume growth condition holds whenever $N \geq \log _{2} C_{d}$, but a smaller $N$ may also suffice; requiring the volume growth condition rather than requiring $N \geq \log _{2} C_{d}$ has the advantage that it is invariant if we replace $d$ by an equivalent metric $d^{\prime}$.

The main result of [15] says that if, for some pair of functions $f, g$ defined on $2 B, g \geq 0$, and some constant $C<\infty$, the following abstract (1,1)-Poincaré inequality is valid:

$$
\text { for all } B^{\prime}=B\left(y, r^{\prime}\right) \subset 2 B: \quad \int_{B^{\prime}}\left|f-f_{B^{\prime}, \lambda}\right| d \lambda \leq C r^{\prime} \int_{B^{\prime}} g d \lambda \text {, }
$$

then for almost every $x \in B$ we have the "representation formula"

$$
C^{\prime}\left|f(x)-f_{B, \lambda}\right| \leq \int_{2 B} \frac{g(y) d(x, y)}{\lambda(B(x, d(x, y)))} d \lambda(y) \equiv G(x),
$$

where $C^{\prime}$ is a constant which depends on $C$ in (6.3), but not otherwise on $f, g$. According to the results in Section 8 of [21], this can be actually be considerably improved: in (6.3), it suffices to assume that $2 B^{\prime} \subset B$ and to replace $\int_{B^{\prime}} g d \lambda$ by $\int_{2 B^{\prime}} g d \lambda$, and we only need to integrate over $B$ rather than over $2 B$ in (6.4). However, we shall work with the (6.4) below.

This representation formula (6.4) is the key to showing that $(\operatorname{Tr})$ and $\left(L^{\infty}\right)$ follow from (6.3) in the above setting. That such implications are possible in 
this general setting is perhaps a little surprising. Even in the Euclidean context with Lebesgue measure, $(1,1)$-Poincaré inequalities are valid on many domains which do not support Trudinger or $L^{\infty}$ imbeddings. For instance, if $\Omega$ is the domain in Example 5.5, then $\Omega$ supports a $(1,1)$-Poincaré inequality since it is star-shaped (as discussed in [4], for example). Since $\Omega$ has a slice property and is not QHBC, it does not support the classical Trudinger inequality and, if the parameter $t$ is larger than some fixed $t_{p}$, then $\Omega$ is not in the correct weak carrot class which (in the presence of the slice condition) is equivalent to the imbedding of $W^{1, p}(\Omega)$ in $L^{\infty}$; see statement (ii) of Section 5 , and the comments following it. It is therefore important that the domains we are considering are metric balls with a doubling measure attached. The above domain $\Omega$ can of course be viewed as a metric ball for Euclidean distance if we simply eliminate the rest of $\mathbb{R}^{n}$ but, although Lebesgue measure restricted to $\Omega$ is doubling, the parameter $N$ is then larger than $n$ (and $N \rightarrow \infty$ as $t \rightarrow \infty$ ).

To prove $\left(L^{\infty}\right)$, we apply Hölder's inequality to (6.4) to get

$$
\left|f(x)-f_{B, \nu}\right| \lesssim \lambda(2 B)\left(f_{2 B} g^{p} d \lambda\right)^{1 / p}\left(f_{2 B} \frac{d(x, y)^{p^{\prime}}}{\lambda(B(x, d(x, y)))^{p^{\prime}}} d \lambda(y)\right)^{1 / p^{\prime}}
$$

We define the annuli $A_{i}=B\left(x, 2^{1-i} r\right) \backslash B\left(x, 2^{-i} r\right), i \geq 0$. For any $1<s<N^{\prime}$, we now use the doubling property of $\lambda$ to get

$$
\begin{aligned}
f_{2 B} \frac{d(x, y)^{s}}{\lambda(B(x, d(x, y)))^{s}} d \lambda(y) & =\frac{1}{\lambda(2 B)} \sum_{i=0}^{\infty} \int_{A_{i}} \frac{d(x, y)^{s}}{\lambda(B(x, d(x, y)))^{s}} d \lambda(y) \\
& \lesssim \frac{r^{s}}{\lambda(2 B)} \sum_{i=0}^{\infty} 2^{-i s} \int_{A_{i}} \lambda\left(B\left(x, 2^{-i} r\right)\right)^{-s} d \lambda(y) \\
& \lesssim \frac{r^{s}}{\lambda(2 B)} \sum_{i=0}^{\infty} 2^{-i(s+N-N s)} \lambda(2 B)^{1-s}
\end{aligned}
$$

and so

$$
f_{2 B} \frac{d(x, y)^{s}}{\lambda(B(x, d(x, y)))^{s}} d \lambda(y) \lesssim \frac{r^{s}}{(s+N-N s) \lambda(2 B)^{s}}
$$

$\left(L^{\infty}\right)$ now follows by taking $s=p^{\prime}$ in (6.6) and combining it with (6.5).

To prove (Tr), we adapt a method of Ziemer [33, Remark 2.8.5]. As we noted at the beginning of Section 3, an Orlicz inequality such as ( $\mathrm{Tr})$ is equivalent to a family of $L^{q}$ inequalities with a certain order of growth on the constants involved, so let us estimate $\int_{2 B} G^{q} d \lambda$ for some $q>2 N$, where $G$ is as in (6.4). We define 
$1<s<N^{\prime}$ by the equation $s^{-1}=N^{\prime-1}+q^{-1}$. Hölder's inequality now implies that

$$
\begin{gathered}
G(x) \leq \lambda(2 B)\left(f_{2 B} \frac{d(x, y)^{s}}{\lambda(B(x, d(x, y)))^{s}} d \lambda(y)\right)^{1 / N^{\prime}}\left(f_{2 B} g^{N} d \lambda\right)^{1 / s^{\prime}} \\
\cdot\left(f_{2 B} \frac{d(x, y)^{s} g^{N}(y)}{\lambda(B(x, d(x, y)))^{s}} d \lambda(y)\right)^{1 / q} \cdot
\end{gathered}
$$

Writing $C_{s}=r /(s+N-N s)^{1 / s} \lambda(2 B)$, we use (6.6) and Fubini's Theorem to get that

$$
f_{2 B} f_{2 B} \frac{d(x, y)^{s} g(y)^{N}}{\lambda(B(x, d(x, y)))^{s}} d \lambda(y) d \lambda(x) \leq C_{s}^{s}\|g\|_{L^{N}\left(2 B, \lambda_{2 B}\right)}^{N}
$$

By this last inequality, (6.6), and (6.7), we get

$$
\begin{aligned}
\|G\|_{L^{q}\left(2 B, \lambda_{2 B}\right)} & \lesssim C_{s}^{s\left(1 / q+1 / N^{\prime}\right)} \lambda(2 B)\|g\|_{L^{N}\left(2 B, \lambda_{2 B}\right)}^{N\left(1 / q+1 / s^{\prime}\right)} \\
& \lesssim r(s+N-N s)^{-1 / s}\|g\|_{L^{N}\left(2 B, \lambda_{2 B}\right)}
\end{aligned}
$$

Letting $q \rightarrow \infty$, and using the fact that $(s+N-N s)^{-1}=q\left(1 / N-1 / N^{2}\right)+$ $1 / N \approx q$, we conclude that

$$
\|G\|_{L^{q}\left(2 B, \lambda_{2 B}\right)} \lesssim q^{1 / N^{\prime}+1 / q} r\|g\|_{L^{N}\left(2 B, \lambda_{2 B}\right)} .
$$

Now, $q^{1 / q}$ has an absolute bound for all $q>N$ and so, using (6.4), the above growth estimate is equivalent to the desired Orlicz inequality.

Several examples falling into this class of examples are mentioned in [15], for example Carnot-Carathéodory spaces defined using Hörmander vector fields or connected Lie groups; in such cases, $g$ is an appropriate horizontal gradient of $f$. There are, however, other examples which are not of Carnot-Carathéodory type and where $g$ is best viewed as an abstract gradient. For example, by the results of [22], we see that (6.3) is true when $X$ is any connected set constructed by glueing a finite number of $N$-dimensional domains along certain subsets (roughly speaking, each glueing subset should have Hausdorff dimension larger than $N-1$ in the neighborhood of each of its points; see the $p=1$ case of [22, Theorem 6.15] for a precise statement). In this situation, the space is locally Euclidean with Lebesgue measure attached, $f$ can be any bounded continuous function, and $g$ any Borel function whose integral over any rectifiable curve in $X$ containing the points $x, y$ is always at least $|f(x)-f(y)|$ (such a function $g$ is termed a "very weak gradient" or "upper gradient" of $f$ ). 


\section{REFERENCES}

[1] B. Bojarski \& T. IwAniec, Analytical foundations of the theory of quasiconformal mappings in $\mathbb{R}^{n}$, Ann. Acad. Sci. Fenn. Ser. A I Math. 8 (1983), 257-324.

[2] S. M. Buckley, Strong doubling conditions, Math. Ineq. Appl. 1 (1998), 533-542.

[3] S. M. Buckley \& P. Koskela, Criteria for Imbeddings of Sobolev-Poincaré type, Internat. Math. Res. Notices (1996), 881-901.

[4] - New Poincaré inequalities from old, Ann. Acad. Sci. Fenn. 23 (1998), 251-260.

[5] - A generalization of Bergman space imbeddings, in preparation.

[6] S. M. Buckley, P. Koskela \& G. Lu, Subelliptic Poincaré inequalities: the case $p<1$, Publ. Mat. 39 (1995), 313-334.

[7] B Boman equals John, Proc. XV Nevanlinna Colloquium, (1996), 91-99, De Gruyter, Berlin.

[8] S. M. Buckley, B. Hanson, \& P. Macmanus, Doubling with respect to general sets, Math. Scand. (to appear).

[9] S. Chanillo ES R. L. Wheeden, Weighted Poincaré and Sobolev inequalities and estimates for the Peano maximal function, Amer. J. Math. 107 (1985), 1191-1226.

[10] R. Colfman 8 G. Weiss, Analyse harmonique non-commutative sur certains espaces homogènes, Lecture notes in Mathematics 242, Springer-Verlag, Berlin, 1971.

[11] D. Edmunds 8 R. Hurri-Syruanen, Weighted Poincaré inequalities and Minkowski content, Proc. Roy. Soc. Edinburgh Sect. A 125 (1995), 817-825.

[12] D. Edmunds \& B. OpIC, Weighted Poincaré and Friedrichs inequalities, J. London Math. Soc. (2) 47 (1993), 79-96.

[13] B. Franchi, C. E. Gutiérrez, 63 R. L. Wheeden, Weighted Sobolev-Poincaré inequalities for Grushin type operators, Comm. P.D.E. 19 (1994), 523-604.

[14] B. Franchi, G. Lu, \& R. L. WheEden, Representation formulas and weighted Poincaré inequalities for Hörmander vector fields, Ann. Inst. Fourier (Grenoble) 45 (1995), 577-604.

[15] _ A relationship between Poincaré-type inequalities and representation formulas in spaces of homogeneous type, Internat. Math. Res. Notices (1996), 1-14.

[16] N. Garofalo \& D.-M. Nhieu, Isoperimetric and Sobolev inequalities for Carnot-Carathéodory spaces and existence of minimal surfaces, Comm. Pure Appl. Math. 49 (1996), 1081-1144.

[17] D. Gilbarg \& N. Trudinger, Elliptic partial differential equations of second or$\operatorname{der}$ (2nd edn), Grundlehren der Mathematischen Wissenschaften 224, Springer-Verlag, Berlin, 1983.

[18] F. W. Gehring \& B. Osgood, Uniform domains and the quasihyperbolic metric, J. Analyse Math. 36 (1979), 50-74.

[19] P. HajŁasz, Sobolev spaces on an arbitrary metric space, Potential Anal. 5 (1996), 403-415.

[20] P. HajŁasz 89 P. Koskela, Sobolev meets Poincaré, C.R. Acad. Sci. Paris 320 (1995), 1211-1215.

[21] Sobolev met Poincaré, preprint.

[22] J. Heinonen $\&$ P. Koskela, Quasiconformal mappings in metric spaces with controlled geometry, Acta Math. 181 (1998), 1-61.

[23] R. Hurri, The weighted Poincaré inequalities, Math. Scand. 67 (1990), 145-160.

[24] P. W. Jones 86 N. G. Makarov, Density properties of harmonic measure, Annals of Math. 142 (1995), 427-455. 
[25] P. Koskela $\&$ S. Rohde, Hausdorff dimension and mean porosity, Math. Ann. 309 (1997), 593-609.

[26] M. Krbec 85 T. Schotт, Embeddings of weighted Sobolev spaces in the borderline case, Real Anal. Exchange 23 (1997/98), 395-420.

[27] M. LApidus, Fractal drum, inverse spectral problems for elliptic operators and partial resolution of the Weyl-Berry conjecture, Trans. Amer. Math. Soc. 325 (1991), 465-529.

[28] O. Martio \& M. Vuorinen, Whitney cubes, p-capacity, and Minkowski content, Expo. Math. 5 (1987), 17-40.

[29] E. SAWYER, Norm inequalities relating singular integrals and the maximal function, Studia Math. 75 (1983), 253-263.

[30] W. Smith \& D. A. Stegenga, Exponential integrability of the quasi-hyperbolic metric on Hölder domains, Ann. Acad. Sci. Fenn. Ser. A I. Math. 16 (1991), 345-360.

[31] - Sobolev imbeddings and integrability of harmonic functions on Hölder domains, Potential Theory (M. Kishi, ed.), de Gruyter, Berlin, 1991.

[32] N. Trudinger, On imbeddings into Orlicz spaces and some applications, J. Math. Mech. 17 (1967), 473-483.

[33] W. P. Ziemer, Weakly differentiable functions, Graduate Texts in Mathematics 120, Springer, New York, 1989.

Acknowledgment. The first author was partially supported by Forbairt.

\author{
Stephen M. Buckley \& Julann O'SheA \\ Department of Mathematics \\ National University of Ireland, Maynooth \\ Co. Kildare, IRELAND \\ E-MAIL: sbuckley@maths.may.ie \\ aoshea@maths.may.ie
}

Submitted: February 26th, 1998; revised: March 18th, 1999. 